\title{
Combined Technology for the Parts Recovery Operating in Stress Factor Conditions
}

\author{
Olena V. Berezshnaya $\mathbb{D D}^{1}{ }^{1}$ Valeriy D. Kassov, ${ }^{1}$ and Eduard P. Gribkov $\mathbb{D}^{2}$ \\ ${ }^{1}$ Handling Systems and Transport Machines Department, Donbass State Engineering Academy, Academichna 72, \\ Kramatorsk 84313, Donetsk Region, Ukraine \\ ${ }^{2}$ Automated Metal Forming Process and Machinery Department, Donbass State Engineering Academy, Academichna 72, \\ Kramatorsk 84313, Donetsk Region, Ukraine \\ Correspondence should be addressed to Olena V. Berezshnaya; elena.kassova07@gmail.com
}

Received 27 September 2019; Accepted 27 February 2020; Published 31 March 2020

Academic Editor: Raffaele Sepe

Copyright (C) 2020 Olena V. Berezshnaya et al. This is an open access article distributed under the Creative Commons Attribution License, which permits unrestricted use, distribution, and reproduction in any medium, provided the original work is properly cited.

\begin{abstract}
The structural factor of samples microplasticity deposited with carbon steel construction tapes in connection with the different sensitivities of the deposited metal to stress concentration was investigated. The most favorable structure of the deposited layer, which is characterized by low sensitivity to stress concentration, is found. The combined technology of parts recovery modes operating in stress factor conditions is given. The covering agent composition, which allows to reduce the energy consumption during heat treatment of the deposited samples after treatment with high-frequency currents, to increase the thermal insulation effect approaching to the furnace treatment conditions, was developed. It was established that retarded cooling in the covering agent of deposited samples after local heating with high-frequency currents provides the increase in the average pressure ferrite during increase in the grain size. It was established that for parts operating under cyclic loading, the structure obtained as a result of combined treatment is the least sensitive to stress concentration.
\end{abstract}

\section{Introduction}

One of the main groups of worn parts, currently being repaired in large quantities, consists of various machine shafts and mechanisms with worn landing and supporting necks, prone to cyclic loads during operation. In most cases, these details limit the resource of machine parts and assemblies. The coefficient of their recovery in the machines complete overhaul is $0.25, \ldots, 0.95$. In terms of increasing the efficiency of worn parts performance restoration, that have small diametrical dimensions and minor losses of the working surface, by increasing them to nominal dimensions with a minimum level of thermodynamic disturbances introduced into the state of the base metal, electrical contact surfacing is successfully used. The process of electrocontact surfacing is carried out by joint deformation of the material being welded and the surface layer of the part heated in the deformation zone by short current pulses. The overlap of the welding points between them is achieved by rotating the part at a speed proportional to the frequency of the current pulses [1-3]. Thus, electric contact surfacing is a combination of heating, deformation, and water cooling operations (the upper layers of the coating are more intensively cooled into a massive foam electrode, and the lower layers are mainly in the part), as a result of that the formation of the deposited layer final structure is characterized by heterogeneity of dispersion and mechanical properties, including different sensitivity to stress concentration [4].

Having determined the optimal structure from the point of view of the surface layers' physical state (deposited layer and heat-affected zone) and sensitivity to stress concentration, one can correctly choose the technology for parts recovery. The regulation of the complex of physical and mechanical indicators (mechanical properties of the surface 
layers, microstructure, residual stresses, and their favorable distribution in the surface layer) is a significant reserve for ensuring the reliability of the recovered parts [5-7]. The presence of an inhomogeneous structure in the surface layer leads to a decrease in the wear resistance of parts operating under stress concentration $[8,9]$. In this regard, it is advisable to conduct research aimed at identifying the microstructure with a low resistance of microplastic deformation and low sensitivity to stress concentration under cyclic loading conditions.

To obtain the specified characteristics of the recovered parts, a stepwise transformation of the properties in a certain sequence with a gradual approximation of the properties to the specified ones [5] is necessary. The staging is conditioned by the fact that, on the one hand, it is impossible to convert all the dissimilar properties of a part with one method at the same time and, on the other hand, to obtain the whole complex of necessary values of the final parameters at one stage [8]. Thus, if electrocontact surfacing with steel tape is aimed at building up the surface layer with the transformation of geometrical parameters of parts and material structure, the subsequent heat treatment is aimed at improving the properties of the surface layer to ensure high stability under cyclic loading conditions. Thus, microplastic deformation plays a decisive role in the concentration of stresses in the deposited layer, and, consequently, affects the stability of the recovered parts operating under cyclic loading conditions. Therefore, the study of the structure and development of the combined recovery technology was carried out regarding the resistance of microplastic deformation.

\section{Prime Novelty Statement}

In this work, the structural factor of samples' microplasticity, deposited with carbon steel construction tapes, was investigated in connection with the different sensitivity of the weld metal to stress concentration.

Based on the conducted studies of the structural factor influence on the sensitivity of the deposited samples to stress concentration under cyclic loading, a combined technology for parts recovery was developed, which includes electrical contact surfacing with structural tapes, subsequent processing by high-frequency currents and retarded cooling in the heat-insulating mixture.

The covering agent composition for the retarded cooling of the deposited samples after treatment with high-frequency currents is proposed. It was established that the introduction of laminated graphite compounds into the covering agent based on silver graphite makes it possible to increase the content of average pressure graphite in the deposited layer by $4.7 \%$ by increasing the thermal insulation effect of the recovered parts approaching the furnace processing conditions.

The results of the study can be used in the development of the technology of electrocontact surfacing of parts from structural steels operating under cyclic loading conditions.

\section{Experimental and Theoretical Studies}

The study of the deposited samples microplasticity was carried out under stretch deformation [10]. Sample preparation included the following operations. Electrocontact welding was made on samples of steel grades C25, $\mathrm{C} 40$, and $530 \mathrm{H} 40$. The material of the tapes was chosen identical to the steel grades of the samples (Table 1). The modes were also selected depending on the steel grade (Tables 2 and 3).

Table 3 shows the weld deposited samples and their chemical composition. After electrocontact surfacing, samples were heated in a laboratory muffle furnace. The deposited samples from steels $\mathrm{C} 25$ and $\mathrm{C} 40$ were subjected to normalization followed by high-temperature tempering (Table 4).

The deposited samples from steel $530 \mathrm{H} 40$ were subjected to heat treatment in several modes, which also determined various structures: they were normalized with tempering (see Table 4), and the deposited samples after quenching (groups II, III) were subjected to tempering at various temperatures to obtain vacation products of different dispersion. After heat treatment, the deposited samples were ground on a flat grinder in a size of $3 \pm 0.1 \mathrm{~mm}$. Grinding was carried out with intensive cooling to eliminate grinding burns. Marking was respectively done considering steel grade and heat treatment group (see Table 4) and samples were made. Tensile tests were carried out on an IM-4R instrument. To study the microstructure in the deformation process, the deposited surface of the sample was polished as a metallographic section, fixed in a special mandrel. To identify the microstructure, chemical etching of microsections in $4 \% \mathrm{HNO}_{3}$ solution in ethyl alcohol was used. To study microplasticity, its homogeneity, depending on the structure along the longitudinal axis of the sample (Figure 1), using a PMT-3 microhardness meter, a number of reference points were drawn with a pyramid under a load of $20 \mathrm{~g}$. Prints were applied every $0.04-0.05 \mathrm{~mm}$, that is, over distances commensurate with the grain size of the deposited samples (Figure 2). According to the measurements of the distances between the prints $a_{\mathrm{K}}$, the change in this distance as a result of deformation was determined:

$$
\Delta a_{k}=a_{k+1}-a_{k} \text {, }
$$

where $a_{k}$ is the distance between points before deformation.

The increase in deformation $\Delta a_{k}$ was positive if the distance between these points increased, and negative if it decreased.

Despite the fact that the study of microplasticity was performed under conditions of static stretching, in some microzones compression deformation was manifested, and in others, stretching deformation was displayed. This indicates a nonuniform deformation of the layer under study. The magnitude of the relative deformation for each base distance was calculated by the formula $\varepsilon_{k}=\ln \left(a_{k+1} / a_{k}\right)$. As it is known [11], this expression is valid for the evaluation of deformation, both in uniaxial stretching and uniaxial compression. Thus, for each given loading, information about microstrain according to $150-160$ values of $\varepsilon_{k}$ was obtained. Next, we determined the average value of the relative deformation of this sign [11]:

$$
\bar{\varepsilon}_{i}=\frac{1}{n} \sum_{k=1}^{n} \varepsilon_{k}
$$


TABLe 1: Metal tapes for electric surfacing.

\begin{tabular}{lccccccccccc}
\hline \multirow{2}{*}{ Steel grade } & \multirow{2}{*}{ Tape thickness $(\mathrm{mm})$} & \multicolumn{9}{c}{ Chemical composition (\%) } \\
& & $\mathrm{C}$ & $\mathrm{Si}$ & $\mathrm{Mn}$ & $\mathrm{Ni}$ & $\mathrm{S}$ & $\mathrm{P}$ & $\mathrm{Cr}$ & $\mathrm{Cu}$ & $\mathrm{Fe}$ & $\mathrm{As}$ \\
\hline $\mathrm{C} 25$ & 0.5 & $0.22-0.3$ & $0.17-0.37$ & $0.5-0.8$ & $<0.25$ & $<0.04$ & $<0.035$ & $<0.25$ & $<0.25$ & $\approx 97$ & $<0.08$ \\
$\mathrm{C} 40$ & 0.5 & $0.37-0.45$ & $0.17-0.37$ & $0.5-0.8$ & $<0.25$ & $<0.035$ & $<0.035$ & $<0.25$ & $<0.3$ & $\approx 97$ & $<0.08$ \\
$530 \mathrm{H} 40$ & 0.5 & $0.36-0.44$ & $0.17-0.37$ & $0.5-0.8$ & $<0.3$ & $<0.035$ & $<0.035$ & $0.8-1.1$ & $<0.3$ & $\approx 97$ & - \\
\hline
\end{tabular}

TABLE 2: Modes of electrocontact surfacing of samples from different steel grades.

\begin{tabular}{lccc}
\hline Steel grade & $I(\mathrm{kA})$ & $\mathrm{P}(\mathrm{kN})$ & $T_{\mathrm{imp}}(\mathrm{sec})$ \\
\hline $\mathrm{C} 25$ & 6.0 & 1.25 & 0.04 \\
$\mathrm{C} 40$ & 6.0 & 1.25 & 0.04 \\
$530 \mathrm{H} 40$ & 6.5 & 1.5 & 0.06 \\
\hline
\end{tabular}

TABLE 3: The chemical composition of the investigated steels.

\begin{tabular}{|c|c|c|c|c|c|c|c|}
\hline \multirow{2}{*}{ Steel grade } & \multicolumn{7}{|c|}{ Elements composition } \\
\hline & $\mathrm{C}$ & $\mathrm{Mn}$ & SI & $\mathrm{Cr}$ & $\mathrm{Ni}$ & S & $\mathrm{P}$ \\
\hline $\mathrm{C} 25$ & 0.23 & 0.29 & 0.17 & Traces & Traces & 0.019 & 0.021 \\
\hline $\mathrm{C} 40$ & 0.41 & 0.32 & 0.21 & Traces & Traces & 0.017 & 0.018 \\
\hline $530 \mathrm{H} 40$ & 0.39 & 0.33 & 0.19 & 0.92 & Traces & 0.016 & 0.019 \\
\hline
\end{tabular}

TABLe 4: Modes of deposited samples heat treatment for microplasticity testing.

\begin{tabular}{lccccccc}
\hline \multirow{2}{*}{ Steel grade } & Group & \multicolumn{3}{c}{ Initial heat treatment mode } & \multicolumn{3}{c}{ Temper mode } \\
& & $T\left({ }^{\circ} \mathrm{C}\right)$ & Soaking time $(\mathrm{h})$ & Cooling medium & $T\left({ }^{\circ} \mathrm{C}\right)$ & Soaking time $(\mathrm{h})$ & Cooling medium \\
\hline C25 & A & $880-900$ & 1 & Air & 620 & 2.5 & With furnace \\
C40 & B & $860-880$ & 1 & With furnace & 620 & 1.5 & With furnace \\
$530 \mathrm{H} 40$ & I & $850-870$ & 1 & With furnace & $640-660$ & 1.5 & With furnace \\
$530 \mathrm{H} 40$ & II & $850-870$ & 1 & Oil & $640-660$ & 1.5 & With furnace \\
$530 \mathrm{H} 40$ & III & $850-870$ & 1 & Oil & $540-560$ & 1.5 & With furnace \\
\hline
\end{tabular}

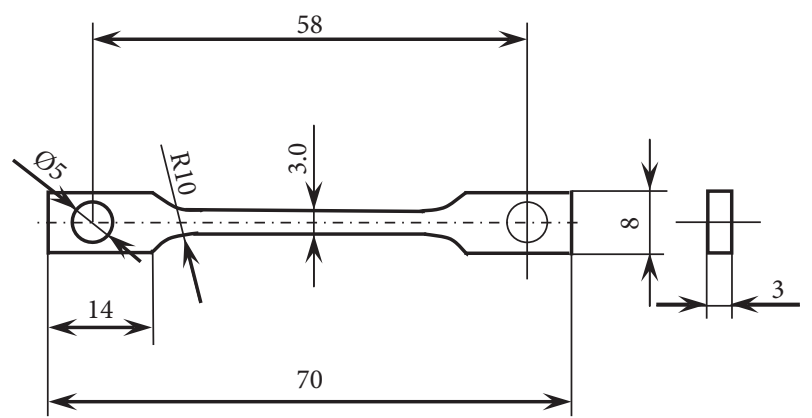

FIgURE 1: Shape and size of samples for microplasticity studies.

where $\bar{\varepsilon}_{i}$ is average relative deformation of the given sign under the influence of the given $i$ loading ( $i$-the loading number); $n$ is the number of measured base distances that determine the deformation of this sign $(\approx 60, \ldots, 70)$.

Dispersion of relative strain:

$$
S^{2}=\frac{1}{n-1}\left[\sum_{k=1}^{n} \varepsilon_{k}^{2}-\frac{1}{n}\left(\sum_{k=1}^{n} \varepsilon_{k}\right)^{2}\right] .
$$

Standard deviation:

$$
S=\sqrt{S^{2}}
$$

The coefficient of microplastic deformation variation:

$$
\gamma=\frac{s}{\varepsilon_{i}} \cdot 100 \%
$$

Experimental results of the microplastic deformation study of the deposited samples in different structural states are given in Tables 5-9. The presented tables show the results of experimental data calculations of the deformation average mathematical value $\bar{\varepsilon}_{i}$, of positive average mathematical value of the strain $+\bar{\varepsilon}_{i}$ and negative $-\bar{\varepsilon}_{i}$ depending on the applied normal stress $\sigma_{i}$. Also the calculated values characterizing the inhomogeneity of the microplastic deformation of the samples deposited by the electrocontact method are shown-the standard deviation $S$ (respectively, according to the total microplastic deformation $S_{1}$, positive $S_{2}$ and negative $S_{3}$ ) and the deformation coefficient according to the total deformation $\gamma_{1}$, the positive deformation $\gamma_{2}$, and negative one $-\gamma_{3}$. 


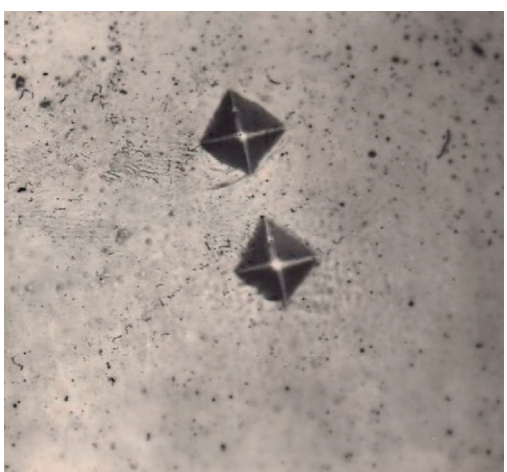

(a)

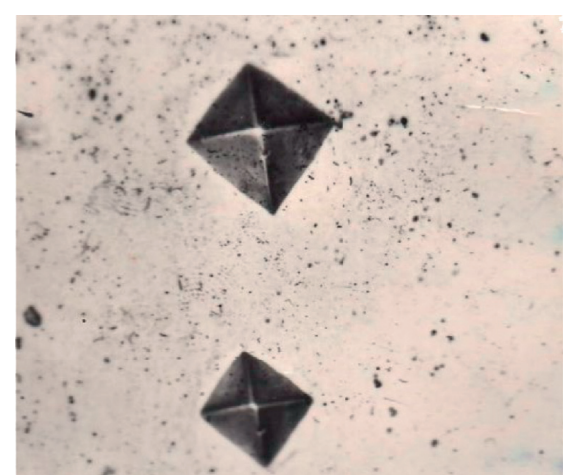

(b)

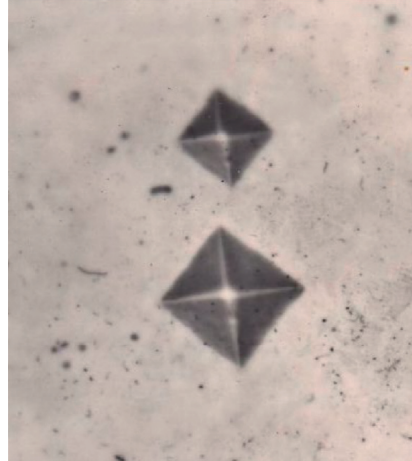

(c)

FIgURE 2: The example of the deposited samples surface microstructure of groups II (a), III (b), and B (c) with applied reference points $(\times 500)$.

Table 5: Experimental data characterizing the microplastic deformation of the deposited samples of steel C25 in the normalized state (group A).

\begin{tabular}{|c|c|c|c|c|c|c|c|c|c|}
\hline \multirow[t]{2}{*}{$\sigma_{i}(\mathrm{MPa})$} & \multicolumn{3}{|c|}{ Deformation } & \multicolumn{3}{|c|}{ Deformation rms deviation } & \multicolumn{3}{|c|}{$\begin{array}{c}\text { Deformation variation coefficient } \\
(\%)\end{array}$} \\
\hline & $\bar{\varepsilon}_{i}$ & $+\bar{\varepsilon}_{i}$ & $-\bar{\varepsilon}_{i}$ & $S_{1}$ & $S_{2}$ & $S_{3}$ & $\gamma_{1}$ & $\gamma_{2}$ & $\gamma_{3}$ \\
\hline 30.5 & 0.009 & 0.005 & 0.009 & 0.008 & 0.011 & 0.006 & 59.45 & 58.71 & 60 \\
\hline 50 & 0.009 & 0.007 & 0.009 & 0.009 & 0.012 & 0.00606 & 76.61 & 72.2 & 60.6 \\
\hline 79.2 & 0.009 & 0.007 & 0.009 & 0.009 & 0.012 & 0.0065 & 80 & 77.42 & 72.2 \\
\hline 109.8 & 0.01 & 0.008 & 0.01 & 0.011 & 0.013 & 0.011 & 81.2 & 100 & 73.3 \\
\hline 140.2 & 0.01 & 0.008 & 0.01 & 0.013 & 0.014 & 0.011 & 83.93 & 105.4 & 78.23 \\
\hline 169.5 & 0.011 & 0.009 & 0.012 & 0.013 & 0.015 & 0.014 & 89.58 & 122.2 & 78.95 \\
\hline 200 & 0.011 & 0.011 & 0.014 & 0.014 & 0.022 & 0.015 & 100 & 150 & 83.78 \\
\hline 219.5 & 0.016 & 0.018 & 0.015 & 0.019 & 0.023 & 0.015 & 100 & 162.5 & 90.79 \\
\hline 250 & 0.047 & 0.054 & 0.019 & 0.030 & 0.039 & 0.017 & 127.2 & 166.6 & 107.1 \\
\hline 280.5 & 0.048 & 0.055 & 0.037 & 0.043 & 0.048 & 0.031 & 137.5 & 170.9 & 122.2 \\
\hline 320 & 0.056 & 0.062 & 0.039 & 0.047 & 0.058 & 0.031 & 144.4 & 209 & 141.6 \\
\hline 341.5 & 0.103 & 0.104 & 0.076 & 0.061 & 0.061 & 0.069 & 172.7 & 220 & 155.5 \\
\hline
\end{tabular}

TABLE 6: Experimental data characterizing the microplastic deformation of deposited samples of steel $530 \mathrm{H} 40$ after annealing (group I).

\begin{tabular}{lccccccccc}
\hline & & Deformation & & \multicolumn{3}{c}{ Deformation rms deviation } & \multicolumn{3}{c}{$\begin{array}{c}\text { Deformation variation } \\
\text { coefficient }(\%)\end{array}$} \\
& $\bar{\varepsilon}_{i}(\mathrm{MPa})$ & $+\bar{\varepsilon}_{i}$ & $-\bar{\varepsilon}_{i}$ & $S_{1}$ & $S_{2}$ & $S_{3}$ & $\gamma_{1}$ & $\gamma_{2}$ & $\gamma_{3}$ \\
\hline 60.7 & 0.015984 & 0.025678 & 0.026 & 0.018 & 0.029 & 0.024 & 59.8 & 59.4 & 50.1 \\
123 & 0.023255 & 0.025846 & 0.034033 & 0.027 & 0.035 & 0.024 & 68.5 & 68.1 & 70.7 \\
186 & 0.030082 & 0.027925 & 0.034880 & 0.033 & 0.042 & 0.028 & 71.9 & 80.3 & 71 \\
244 & 0.032564 & 0.030556 & 0.035800 & 0.041 & 0.045 & 0.029 & 79.8 & 112.9 & 73.9 \\
305 & 0.033239 & 0.035679 & 0.036192 & 0.041 & 0.045 & 0.029 & 81.2 & 125.3 & 80.1 \\
366 & 0.039689 & 0.042874 & 0.037646 & 0.048 & 0.047 & 0.029 & 101.3 & 137.4 & 81.2 \\
427 & 0.041734 & 0.045840 & 0.039218 & 0.084 & 0.075 & 0.0306 & 131.1 & 235.6 & 92.3 \\
488 & 0.051 & 0.056 & 0.041 & 0.088 & 0.103 & 0.032 & 201.2 & 240.7 & 238 \\
528 & 0.057 & 0.066 & 0.043296 & 0.216 & 0.108 & 0.081 & 322.7 & 290.1 & 466.5 \\
538 & 0.066929 & 0.079 & 0.044793 & 0.2780 & 0.187 & 0.209 & 500.5 & 302.2 & 487.2 \\
602 & 0.070 & 0.101894 & 0.045 & 0.6305 & 0.308 & 0.303 & 705.4 & 524.1 & 868.6 \\
\hline
\end{tabular}

The experimental studies results of the samples deposited by the electrocontact method microplasticity in the form of a graph are presented in Figure 3.

For processing experimental data of the deposited samples microplasticity studies were carried out by mathematical modeling in the STATISTICA StatSoft 10 software environment. For each series of experimental data, as a result of modeling using the STATISTICA StatSoft 10 software environment, basic statistics were determined for the values of microplastic deformation and stress (Table 10).

The verification of the stress sampling under the tension of the deposited samples on the belonging to the normal 
TABLE 7: Experimental data characterizing the microplastic deformation of the deposited samples of steel $530 \mathrm{H} 40$ after heat treatment according to mode II (group II).

\begin{tabular}{|c|c|c|c|c|c|c|c|c|c|}
\hline \multirow[t]{2}{*}{$\sigma_{i}(\mathrm{MPa})$} & \multicolumn{3}{|c|}{ Deformation } & \multicolumn{3}{|c|}{ Deformation rms deviation } & \multicolumn{3}{|c|}{$\begin{array}{l}\text { Deformation variation } \\
\text { coefficient }(\%)\end{array}$} \\
\hline & $\bar{\varepsilon}_{i}$ & $+\bar{\varepsilon}_{i}$ & $-\bar{\varepsilon}_{i}$ & $S_{1}$ & $S_{2}$ & $S_{3}$ & $\gamma_{1}$ & $\gamma_{2}$ & $\gamma_{3}$ \\
\hline 59 & 0.007 & 0.005313 & 0 & 0.006 & 0.0056 & 0 & 25 & 0 & 0 \\
\hline 119 & 0.008 & 0.006 & 0.001493 & 0.006 & 0.00604 & 0 & 69 & 70 & 0 \\
\hline 188 & 0.008329 & 0.008 & 0.008 & 0.011 & 0.007 & 0.000504 & 75 & 70 & 63 \\
\hline 240.2 & 0.010599 & 0.009175 & 0.009 & 0.013 & 0.0173 & 0.0067 & 80 & 89.8 & 68 \\
\hline 300 & 0.010668 & 0.009833 & 0.009091 & 0.021 & 0.018 & 0.011 & 85 & 100 & 74 \\
\hline 359 & 0.0133301 & 0.013591 & 0.010165 & 0.021 & 0.021 & 0.017 & 122 & 117 & 96 \\
\hline 419 & 0.016631 & 0.016632 & 0.013132 & 0.023 & 0.023 & 0.021 & 132 & 131 & 122 \\
\hline 481 & 0.017676 & 0.019566 & 0.016879 & 0.024 & 0.024 & 0.022 & 135 & 150 & 167 \\
\hline 537 & 0.0226523 & 0.025 & 0.017148 & 0.027 & 0.025 & 0.023 & 154 & 183 & 207 \\
\hline 601 & 0.026 & 0.030271 & 0.023797 & 0.035 & 0.034 & 0.035 & 162 & 250 & 266 \\
\hline 659 & 0.032868 & 0.033985 & 0.024 & 0.036 & 0.045 & 0.035 & 196 & 490 & 1008 \\
\hline 718 & 0.055 & 0.065 & 0.032216 & 0.055 & 0.055 & 0.038 & 270 & 571 & 2545 \\
\hline
\end{tabular}

TABLE 8: Experimental data characterizing the microplastic deformation of deposited samples of steel $530 \mathrm{H} 40$ after heat treatment according to mode III (group III).

\begin{tabular}{|c|c|c|c|c|c|c|c|c|c|}
\hline \multirow[t]{2}{*}{$\sigma_{i}(\mathrm{MPa})$} & \multicolumn{3}{|c|}{ Deformation } & \multicolumn{3}{|c|}{ Deformation rms deviation } & \multicolumn{3}{|c|}{$\begin{array}{l}\text { Deformation variation } \\
\text { coefficient }(\%)\end{array}$} \\
\hline & $\bar{\varepsilon}_{i}$ & $+\bar{\varepsilon}_{i}$ & $-\bar{\varepsilon}_{i}$ & $S_{1}$ & $S_{2}$ & $S_{3}$ & $\gamma_{1}$ & $\gamma_{2}$ & $\gamma_{3}$ \\
\hline 201 & 0.027 & 0.026 & 0.011 & 0.013 & 0.017 & 0 & 47 & 60 & 0 \\
\hline 249 & 0.03 & 0.026 & 0.027 & 0.017 & 0.018 & 0.017 & 69 & 65.4 & 48.6 \\
\hline 361 & 0.031 & 0.027 & 0.031 & 0.022 & 0.018 & 0.018 & 70 & 65.5 & 53.1 \\
\hline 400 & 0.031 & 0.029 & 0.032 & 0.022 & 0.019 & 0.021 & 75 & 69.2 & 65.63 \\
\hline 449 & 0.031 & 0.03 & 0.032 & 0.024 & 0.024 & 0.025 & 75 & 73 & 69.63 \\
\hline 500 & 0.032 & 0.03 & 0.032 & 0.024 & 0.025 & 0.025 & 77.4 & 73 & 75.7 \\
\hline 549 & 0.033 & 0.031 & 0.035 & 0.024 & 0.026 & 0.027 & 78 & 78 & 77.1 \\
\hline 604 & 0.036 & 0.032 & 0.036 & 0.025 & 0.028 & 0.028 & 79 & 86 & 80.9 \\
\hline 645 & 0.039 & 0.04 & 0.037 & 0.031 & 0.033 & 0.029 & 80 & 88 & 89.6 \\
\hline 659 & 0.041 & 0.045 & 0.037 & 0.037 & 0.043 & 0.0302 & 88 & 90.3 & 94.3 \\
\hline 748 & 0.087 & 0.089 & 0.042 & 0.066 & 0.065 & 0.034 & 94 & 107 & 107.4 \\
\hline
\end{tabular}

TABLE 9: Experimental data characterizing the microplastic deformation of the deposited samples of steel C40 after normalization (group B).

\begin{tabular}{|c|c|c|c|c|c|c|c|c|c|}
\hline \multirow[t]{2}{*}{$\sigma_{i}(\mathrm{MPa})$} & \multicolumn{3}{|c|}{ Deformation } & \multicolumn{3}{|c|}{ Deformation rms deviation } & \multicolumn{3}{|c|}{$\begin{array}{c}\text { Deformation variation coefficient } \\
(\%)\end{array}$} \\
\hline & $\bar{\varepsilon}_{i}$ & $+\bar{\varepsilon}_{i}$ & $-\bar{\varepsilon}_{i}$ & $S_{1}$ & $S_{2}$ & $S_{3}$ & $\gamma_{1}$ & $\gamma_{2}$ & $\gamma_{3}$ \\
\hline 151 & 0.02 & 0.026 & 0.01 & 0.031 & 0.0301 & 0.013 & 65.77 & 54.8 & 47.50 \\
\hline 200 & 0.025 & 0.032 & 0.014 & 0.032 & 0.034 & 0.013 & 71.4 & 63.48 & 59.3 \\
\hline 251 & 0.028 & 0.032 & 0.016 & 0.032 & 0.035 & 0.016 & 91.4 & 83.3 & 92.9 \\
\hline 301 & 0.029 & 0.035 & 0.016 & 0.036 & 0.039 & 0.018 & 100 & 86 & 94.7 \\
\hline 351 & 0.031 & 0.035 & 0.017 & 0.037 & 0.0403 & 0.019 & 103.2 & 94.4 & 96.08 \\
\hline 401 & 0.031 & 0.035 & 0.019 & 0.037 & 0.0403 & 0.0201 & 127.6 & 115.14 & 125.6 \\
\hline 452 & 0.035 & 0.042 & 0.02 & 0.043 & 0.044 & 0.029 & 144 & 125.9 & 130 \\
\hline 502 & 0.07 & 0.084 & 0.041 & 0.05 & 0.046 & 0.035 & 153.6 & 137.5 & 145 \\
\hline 533 & 0.111 & 0.115 & 0.051 & 0.073 & 0.073 & 0.049 & 185 & 150 & 225 \\
\hline
\end{tabular}

distribution law was performed using the Kolmogorov-Smirnov criterion (Figures $4-8$ and Tables 11 and 12).

The calculated values of the Kolmogorov-Smirnov criterion at the 5\% significance level for all groups of samples do not exceed the critical $(d=1.358)$. The calculation of the distribution by the Lilliefors criterion confirms the conformity of the distribution to the null hypothesis (for the significance level of 0.05 , the critical value of the Lillieffors criterion is 0.886 ). The graphs of the normal distribution of the studied characteristics have a slight shift, according to the change in the values of the asymmetry coefficient, and are distinguished by a gentle curve. Thus, the obtained 


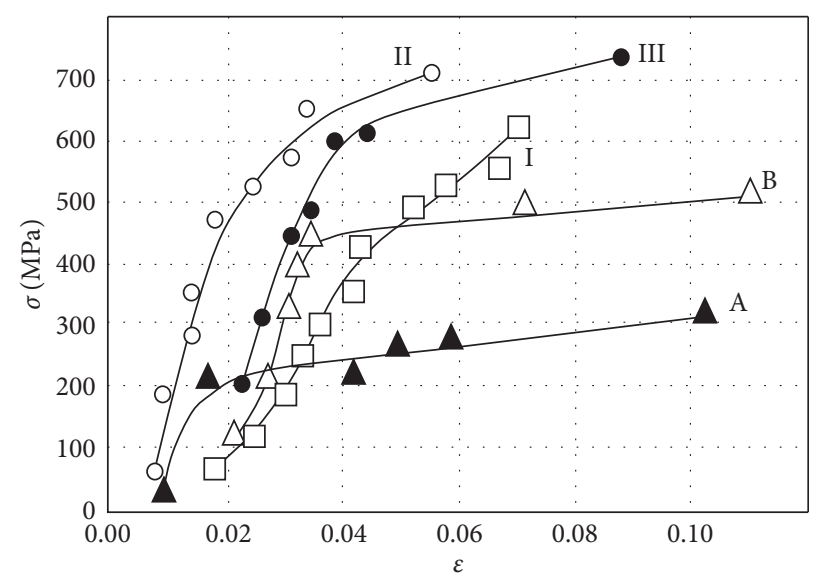

Figure 3: Experimental data on the change in the value of microplastic deformation $\varepsilon$ in the deposited samples after heat treatment (according to Table 4) with increasing stress $\sigma$.

TABLE 10: The results of experimental data statistical processing.

\begin{tabular}{lcccc}
\hline Group & Parameter & Sample average & Minimum value & Maximum value \\
\hline \multirow{2}{*}{ A } & $\varepsilon_{i}$ & 0.0297 & 0.00900 & 0.1030 \\
& $\sigma_{i}$ & 182.5583 & 30.50000 & 341.5000 \\
I & $\varepsilon_{i}$ & 0.0334 & 0.0160 & 0.0510 \\
& $\sigma_{i}$ & 279.9625 & 60.7000 & 488.0000 \\
II & $\varepsilon_{i}$ & 0.0191 & 0.0070 & 0.0550 \\
& $\sigma_{i}$ & 373.3500 & 59.0000 & 659.0000 \\
III & $\varepsilon_{i}$ & 0.0314 & 0.0270 & 0.0360 \\
& $\sigma_{i}$ & 414.1250 & 201.0000 & 604.0000 \\
B & $\varepsilon_{i}$ & 0.0336 & 0.0200 & 0.0700 \\
& $\sigma_{i}$ & 326.1250 & 151.0000 & 502.0000 \\
\hline
\end{tabular}

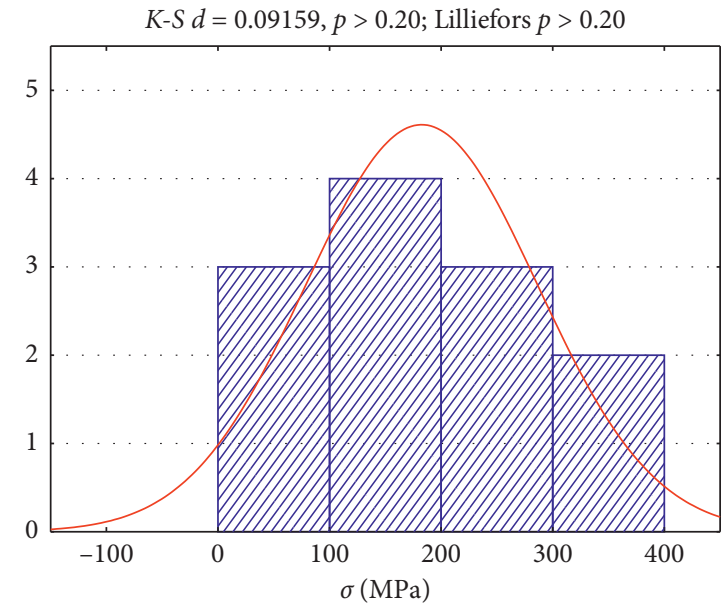

— Expected normal

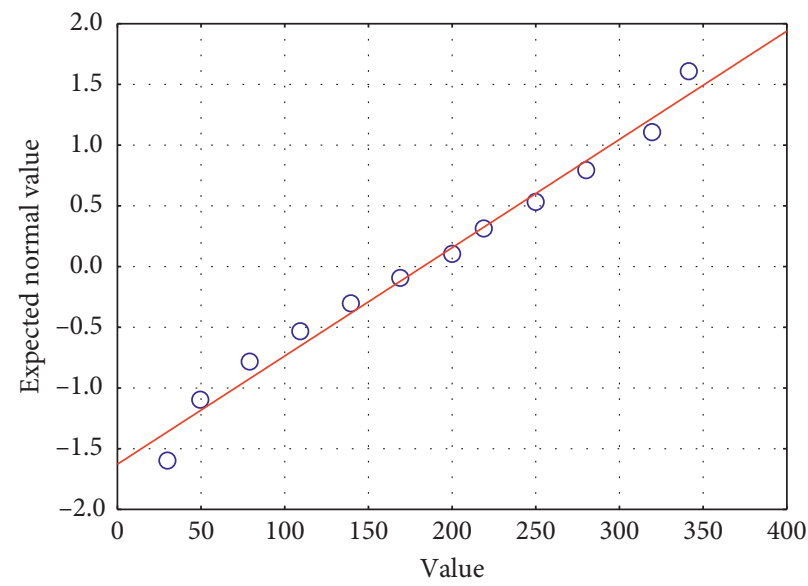

(b)

FIgURE 4: The distribution histogram of the stress experimental values with the overlaid curve of the expected normal distribution (a) and the graph of the normal distribution (b) for the deposited samples of group A.

histograms of the distributions, including the values of the asymmetry and kurtosis coefficients, indicate that the experimental data correspond to the normal distribution law.
To determine the statistical dependencies of changes in the experimental data, approximation by an exponential function was applied (Tables 13 and 14). 


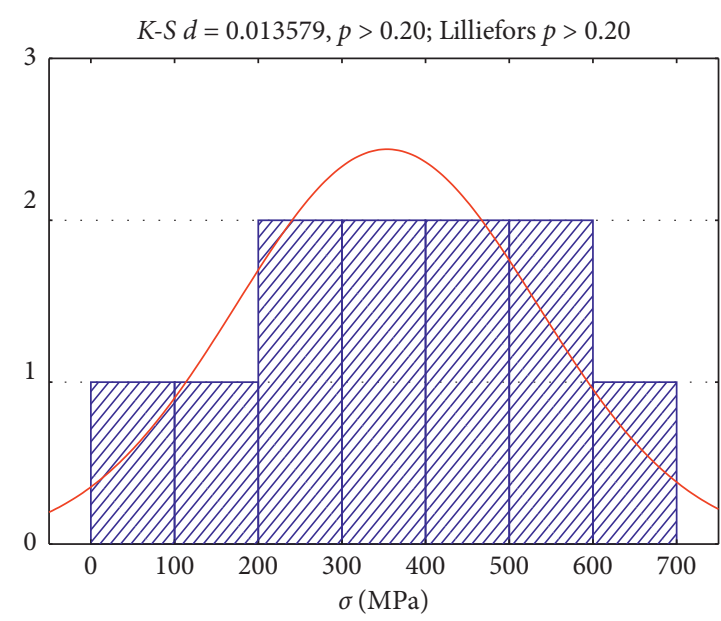

— Expected normal

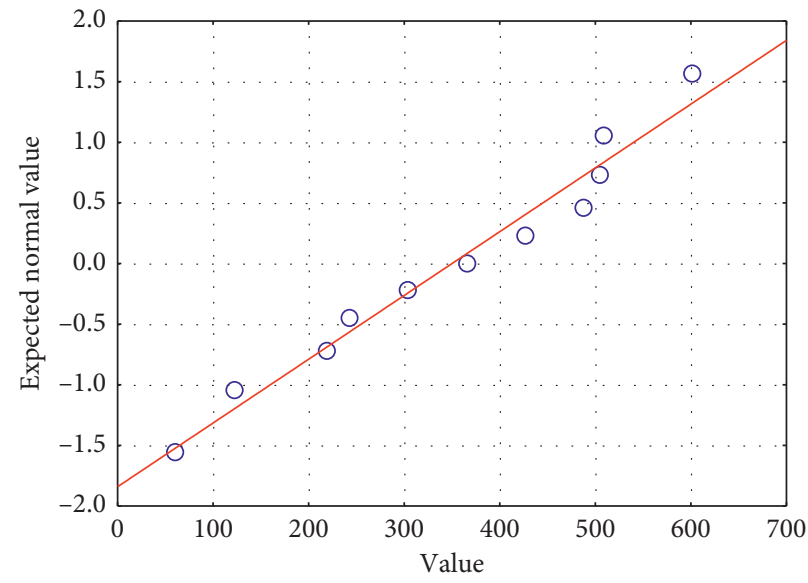

(b)

FIGURE 5: The distribution histogram of the stress experimental values with the superimposed curve of the expected normal distribution (a) and the graph of the normal distribution (b) for the deposited samples of group I.

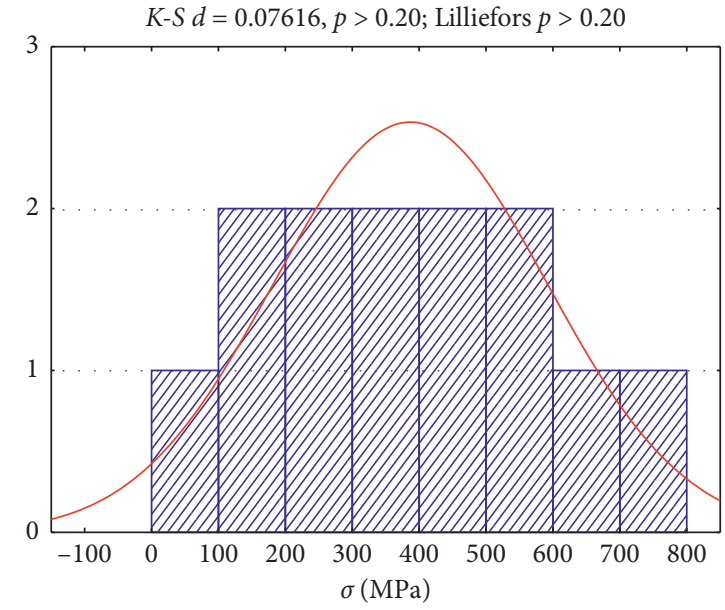

— Expected normal

(a)

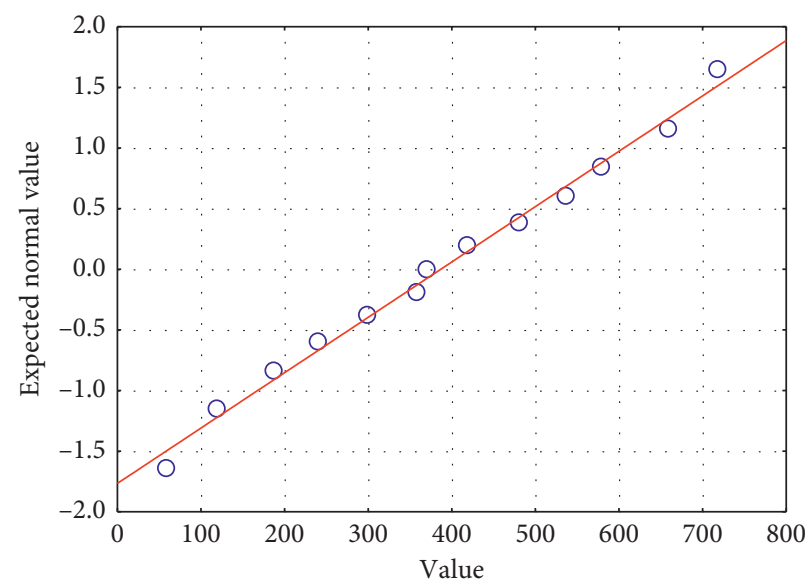

(b)

FiguRE 6: The distribution histogram of the stress experimental values with the superimposed curve of the expected normal distribution (a) and the graph of the normal distribution (b) for the deposited samples in group II.

By the given equations, the smallest stresses are calculated, causing the first acts of microplastic deformation $\sigma_{\text {critical }}$. According to the calculated statistical equations, graphical dependence was constructed, demonstrating the nature of the change in the values of microplastic deformation with increasing applied tensile stress (Figures 9-13).

According to the obtained data (see Table 14) and the change in graphical dependencies (see Figures 9-13), it may be assumed that regression equations have a rather high correlation coefficient. Depending on the sample group under study, statistical models explain from 74 to $90 \%$ of the initial data. Given the low significance level of the Fisher criterion, on average, with an $80 \%$ confidence level, it is arguable that the obtained models are adequate.

Studies of the microstructure of the deposited samples after heat treatment according to the indicated modes showed that samples of group A acquired a ferrite-pearlite structure after normalization, samples of group B after normalization and samples of group I after annealing acquired a pearlite-ferritic structure, samples of group II after thermal improvement by the mode II are characterized by a sorbitol structure, and samples of group III, after thermoirradiation with mode III, are troostite. In the study of microplastic deformation, residual microstrain appeared 


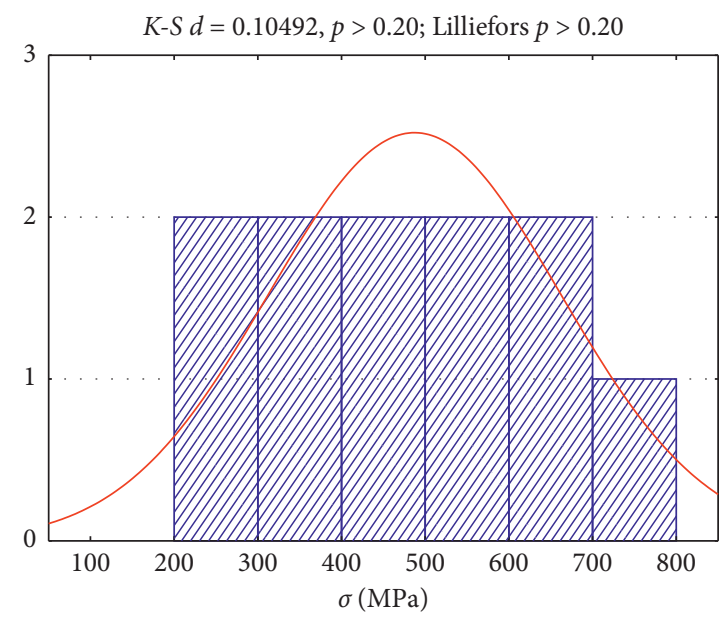

— Expected normal

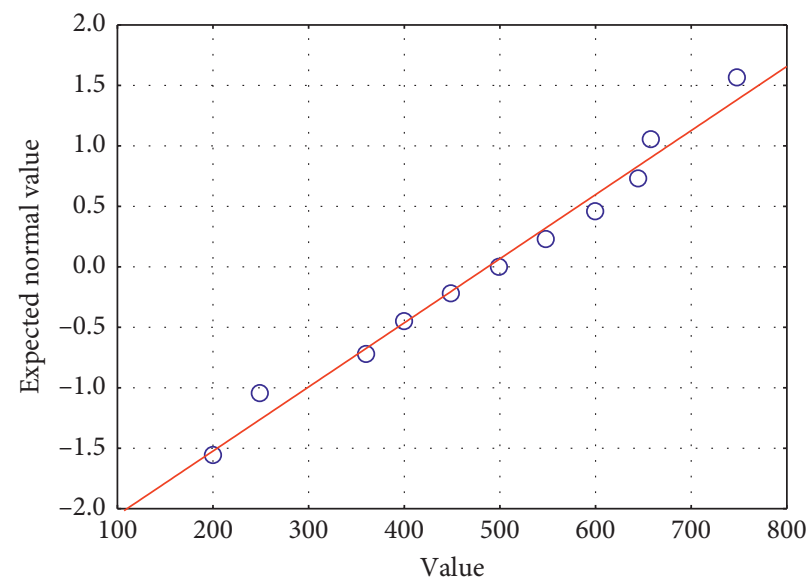

(b)

FIGURE 7: The distribution histogram of the stress experimental values with the superimposed curve of the expected normal distribution (a) and the graph of the normal distribution (b) for the deposited samples of group III.

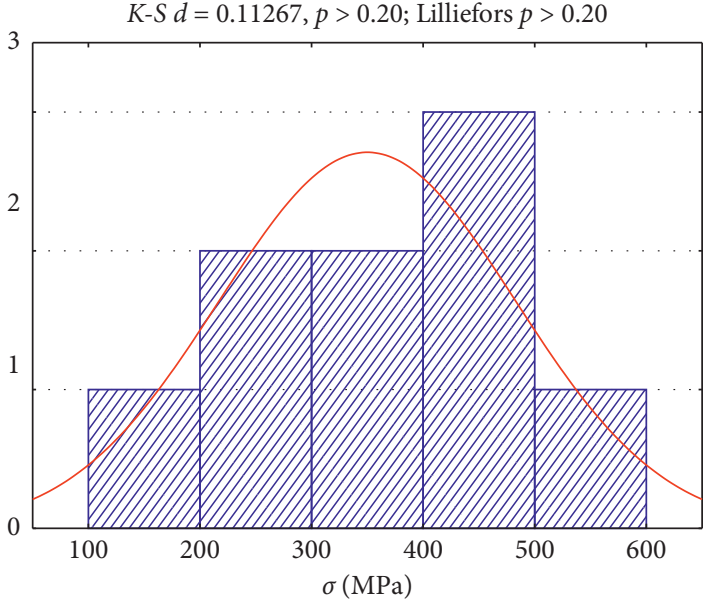

— Expected normal

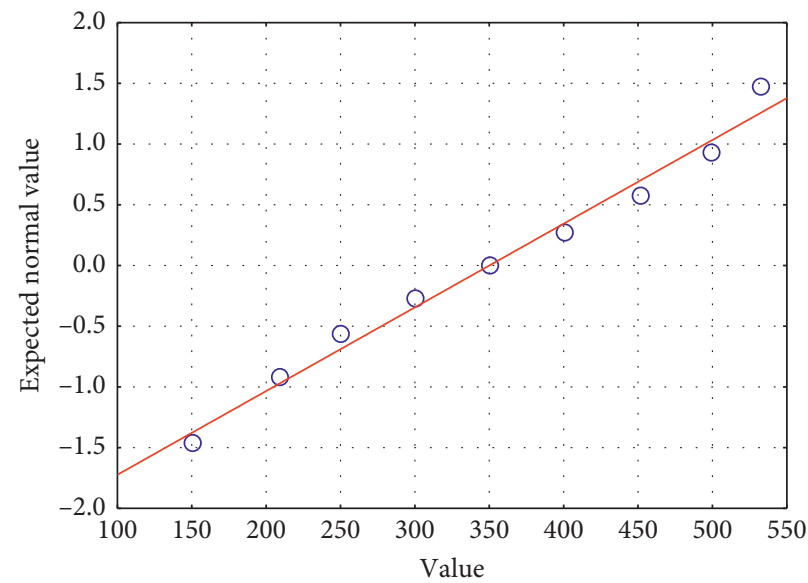

(b)

Figure 8: The distribution histogram of the stress experimental values with the overlaid curve of the expected normal distribution (a) and the graph of the normal distribution (b) for the deposited samples of group B.

TABLE 11: The results of checking the sample for normality by Kolmogorov-Smirnov and Lilliefors tests.

\begin{tabular}{lccc}
\hline Group & $\begin{array}{c}\text { Kolmogorov-Smirnov } \\
\text { test, } d\end{array}$ & $\begin{array}{c}\text { Level of } \\
\text { significance, } p\end{array}$ & $\begin{array}{c}\text { Lilliefors } \\
\text { test, } p\end{array}$ \\
\hline A & 0.09159 & $>0.20$ & $>0.20$ \\
I & 0.13579 & $>0.20$ & $>0.20$ \\
II & 0.07616 & $>0.20$ & $>0.20$ \\
III & 0.10492 & $>0.20$ & $>0.20$ \\
B & 0.11267 & $>0.20$ & $>0.20$ \\
\hline
\end{tabular}

even at the lowest stresses. It is typical that these stresses increase with an increase in the volume content of perlite and simultaneously with a decrease in the tempering
TABLE 12: The values of the asymmetry and excess coefficients.

\begin{tabular}{lcc}
\hline Group & Asymmetry coefficient & Coefficient of excess \\
\hline A & 0.048806 & -1.18870 \\
I & -0.239882 & -1.27903 \\
II & -0.004958 & -1.18292 \\
III & -0.267814 & -0.870051 \\
B & -0.074895 & -1.30778 \\
\hline
\end{tabular}

temperature during thermal improvement. Thus, if the deposited sample contains a relatively large volume of excess ferrite grains (samples of group A), then fewer obstacles occur to the dislocation slip, and, respectively, the stress for the initial microplastic deformation decreases. 
TABLE 13: Equations that approximate the relationship between stress and total microplastic deformation of the deposited samples after various heat treatments.

\begin{tabular}{lccccc}
\hline Surfacing metal & Type of heat treatment & Group & Structure & Equation $\sigma_{i}=f\left(\varepsilon_{i}\right)$ & $\sigma_{\text {calc }}(\mathrm{MPa})$ \\
\hline $\mathrm{C} 25$ & Normalization & A & Ferrite + perlite & $\sigma_{i}=253.250+5540.243 \varepsilon_{i}-\left(5.017 / \varepsilon_{i}\right)$ & 30.56 \\
$\mathrm{C} 40$ & Normalization & B & Perlite + ferrite & $\sigma_{i}=953.72-3160.09 \varepsilon_{i}-\left(15.59 / \varepsilon_{i}\right)$ & 111.02 \\
$530 \mathrm{H} 40$ & Annealing & I & Perlite + ferrite & $\sigma_{i}=336.258+2540.24 \varepsilon_{i}-\left(8.017 / \varepsilon_{i}\right)$ & 57.35 \\
$530 \mathrm{H} 40$ & Thermal improvement in mode II & II & Sorbitol & $\sigma_{i}=501.968+4323.546 \varepsilon_{i}-\left(2.817 / \varepsilon_{i}\right)$ & 79.804 \\
$530 \mathrm{H} 40$ & Thermal improvement in mode III & III & Troostit & $\sigma_{i}=1767.33-1117.25 \varepsilon_{i}-\left(41.31 / \varepsilon_{i}\right)$ & 207.16 \\
\hline
\end{tabular}

TABLE 14: Regression calculation results.

\begin{tabular}{|c|c|c|c|c|c|}
\hline Group & Equation $\sigma_{i}=f\left(\varepsilon_{i}\right)$ & $R$ & $R^{2}$ & $F$ & $p$ \\
\hline $\mathrm{A}$ & $\sigma_{i}=253.250+5540.243 \varepsilon_{i}-\left(5.017 / \varepsilon_{i}\right)$ & 0.96 & 0.93 & 61.5 & 0.0006 \\
\hline $\mathrm{B}$ & $\sigma_{i}=953.72-3160.09 \varepsilon_{i}-\left(15.59 / \varepsilon_{i}\right)$ & 0.93 & 0.87 & 17.25 & 0.00066 \\
\hline I & $\sigma_{i}=336.258+2540.24 \varepsilon_{i}-\left(8.017 / \varepsilon_{i}\right)$ & 0.96 & 0.93 & 49.4 & 0.000074 \\
\hline II & $\sigma_{i}=501.968+4323.546 \varepsilon_{i}-\left(2.817 / \varepsilon_{i}\right)$ & 0.86 & 0.74 & 12.64 & 0.0024 \\
\hline III & $\sigma_{i}=1767.33-1117.25 \varepsilon_{i}-\left(41.31 / \varepsilon_{i}\right)$ & 0.95 & 0.90 & 44.59 & 0.000021 \\
\hline
\end{tabular}

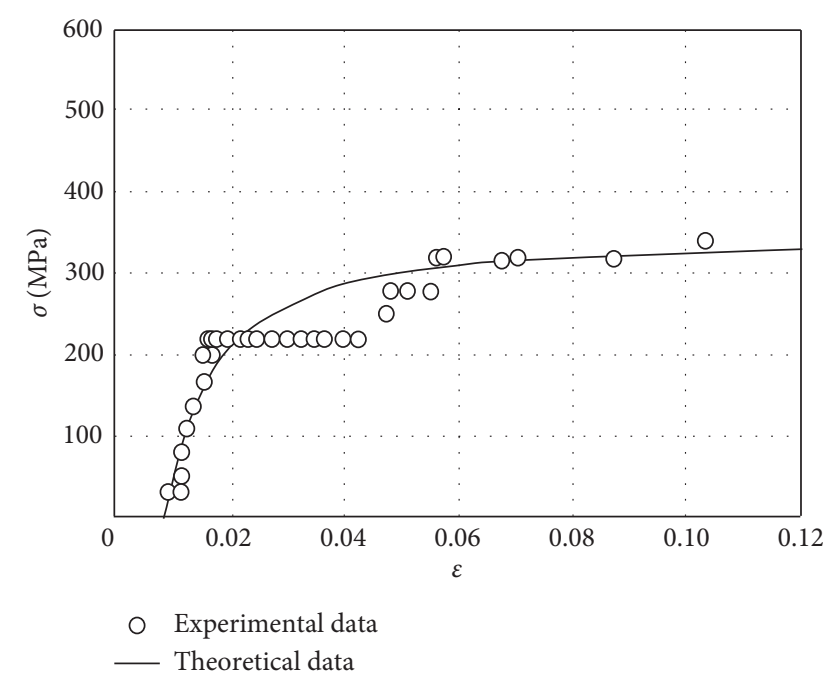

Figure 9: The dependence between the average value of microplastic deformation and the applied tensile stress in the deposited samples of steel C25 in the normalized state (group A).

In sorbitol structures with a developed intergranular surface, there are many more obstacles for sliding, which causes sensitivity to stress concentration. Deposited samples of steel C25 require the least stress for microplastic deformation. In terms of the study, these stresses are $30.5 \mathrm{MPa}$. In the deposited samples with a high content in the structure of cementite, these stresses are higher. The highest stresses occur in samples that, after surfacing, were subjected to thermal enhancement to sorbitol and tempering troostite [5]. Only in this structural state, the samples are the most sensitive to the stress concentration under cyclic loading conditions.

It is recommended [11] to use either high tempering at $620, \ldots, 640^{\circ} \mathrm{C}$, or normalization with high tempering as heat treatment of deposited joints for medium carbon steels. As a result of this heat treatment, a fine sorbitol structure is

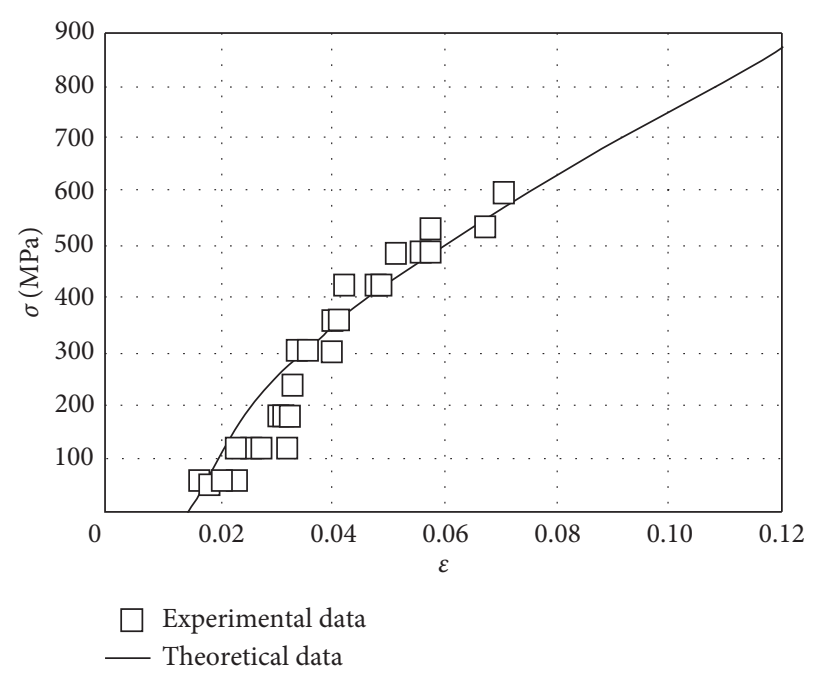

Figure 10: The dependence between the average value of microplastic deformation and the applied tensile stress in the deposited samples of steel 530H40 after annealing (group I).

formed, the hardness decreases, and the mechanical properties are increased. However, as was established in the course of the experiments, for parts operating under cyclic loading, such a structure is most sensitive to stress concentration. It was also found that the deposited samples containing excess ferrite in the structure have less resistance to microplastic deformation than samples with the structure of sorbitol tempering.

When choosing the mode and method of heat treatment, it is necessary to take into account the fact that not the whole product is exposed to the restoration by surfacing, but only worn areas, and the tape thickness and the depth of the heataffected zone do not exceed a few millimeters [12, 13]. Therefore, the most optimal in this case will be the use of the local surface method of heating by high-frequency currents $[14,15]$. 


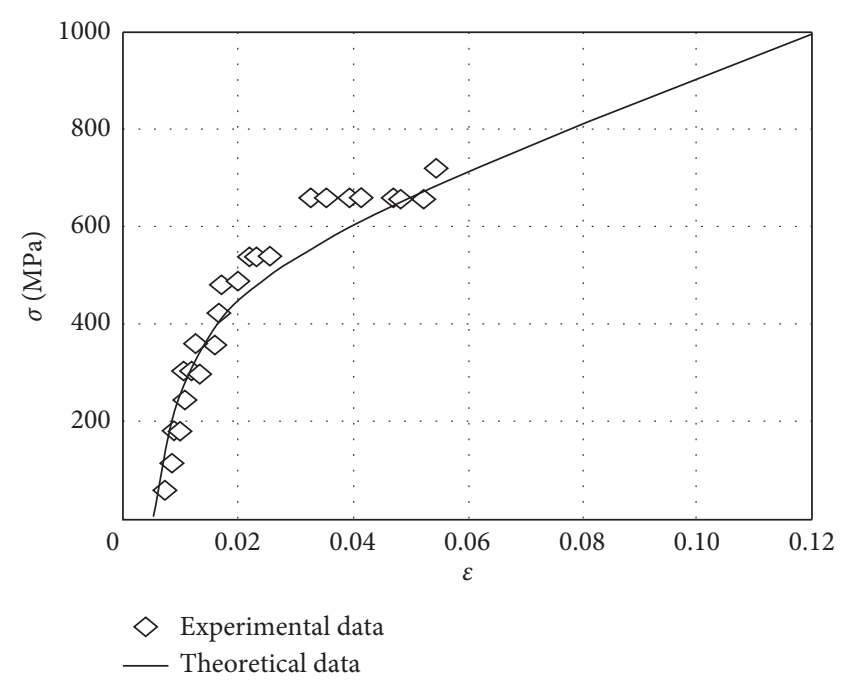

FIGURE 11: The dependence between the average value of microplastic deformation and the applied tensile stress in the deposited samples of steel $530 \mathrm{H} 40$ after thermal improvement in mode II (group II).

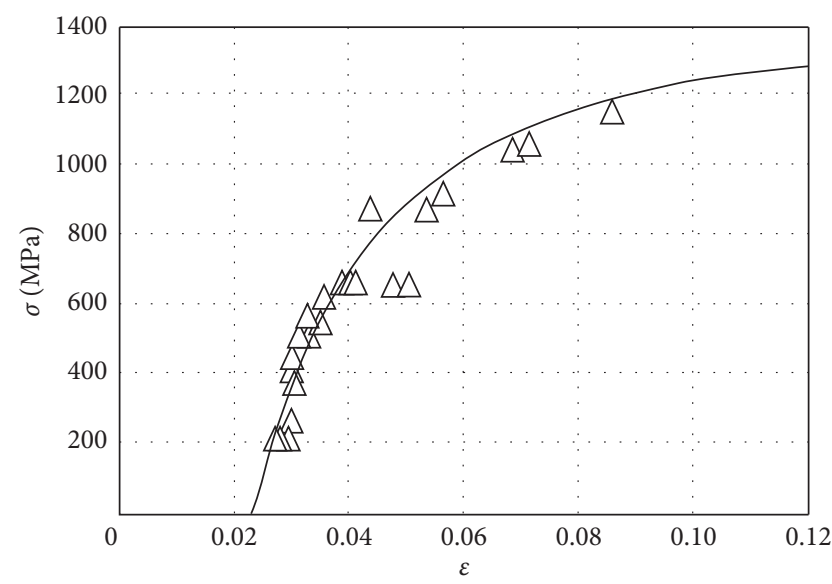

$\triangle$ Experimental data

- Theoretical data

Figure 12: The dependence between the average value of microplastic deformation and the applied tensile stress in the deposited samples of steel $530 \mathrm{H} 40$ after thermoenhancement in mode III (group III).

The structure after high-frequency normalization differs from the structure after normalization from furnace heating [15]. In a conventional oven-heated normalization, the structure consists of pearlite grains evenly distributed between the ferrite grains. Both structural parts are represented in the form of rather large grains. With high-frequency heating, the total amount of free ferrite in the structure is somewhat less than with a conventional furnace. The decrease in the number of structurally free ferrite is obviously explained by the fact that after heating at high speed, which leads to the formation of fine-grained austenite, the release of ferrite during cooling is rather difficult. In perlite, more ferrite remains than is necessary for the formation of a

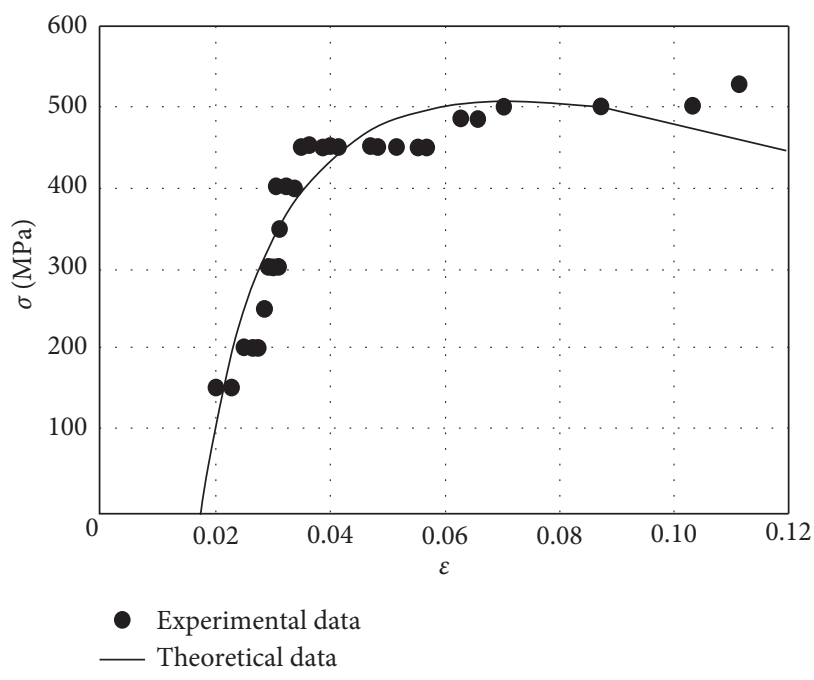

FIgURE 13: The dependence between the average value of microplastic deformation and the applied tensile stress in the deposited samples of steel C40 after normalization (group B).

eutectoid. In some areas, thinner perlite, which is similar in structure to sorbitol, may be formed. In addition, cooling in air leads to the formation of scale, which requires an increase in allowances for mechanical processing, and, consequently, an increase in the thickness of the deposited tape. In order to eliminate these drawbacks and obtain the necessary structure, cooling after induction heating should be carried out in an environment that would provide a slower cooling rate and would prevent surface oxidation.

According to [16], the temperature and duration of the pearlite transformation depend on the rate of the steel cooling from the austenitization temperature to the temperature at which the pearlite transformation begins. Therefore, the higher the cooling rate, the lower the conversion temperature and the shorter its duration. For all cooling conditions (with a furnace, in air, and in graphite (Figure 14)) [17], four stages are typical: (1) cooling from the austenitization temperature to the temperature at which ferrite is released; (2) cooling from the temperature of the onset of ferrite extraction to the onset of pearlite conversion; (3) pearlite transformation itself; (4) cooling from the temperature of the end of the perlite transformation to a temperature of $200^{\circ} \mathrm{C}$.

The resulting character of the change in the structure is associated primarily with the cooling rate of the samples prior to the start of the pearlite transformation. An increase in the cooling rate will lead to a decrease in the pearlite transformation temperature and a decrease in its duration. In all cases, the pearlite transformation occurs at a constant temperature, that is, has an isothermal character. This indicates that pearlite transformation occurs due to its own internal energy. Since this energy compensates for the losses to the environment, the transformation duration depends on the temperature of the cooling medium.

At a lower supercooling temperature, the transformation quickly ends due to the fact that the internal heat is intensively spent on heating the environment, and therefore 


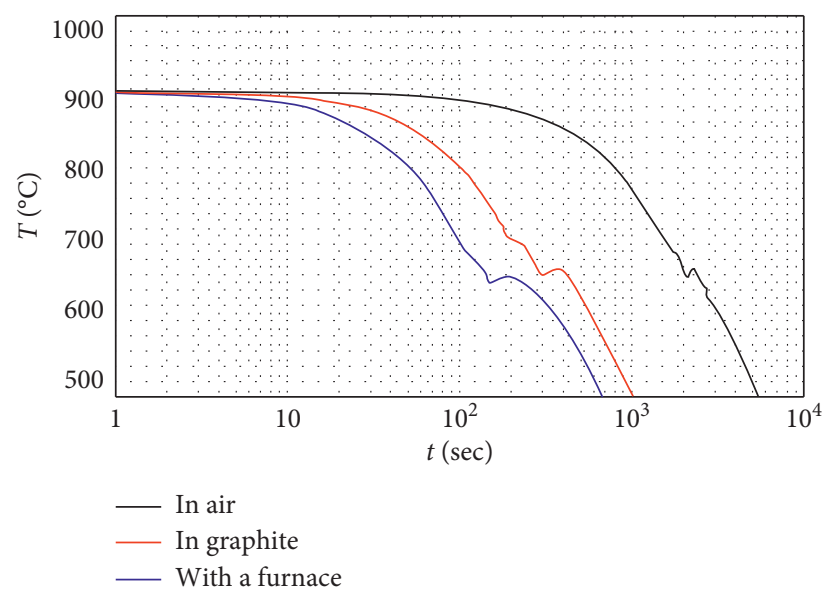

Figure 14: Temperature change of deposited steel C45 samples with a $20 \mathrm{~mm}$ diameter under various cooling conditions after heat treatment [17].

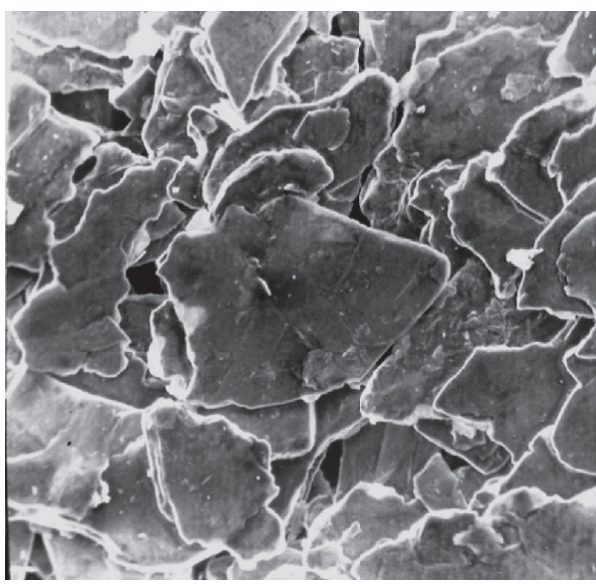

(a)

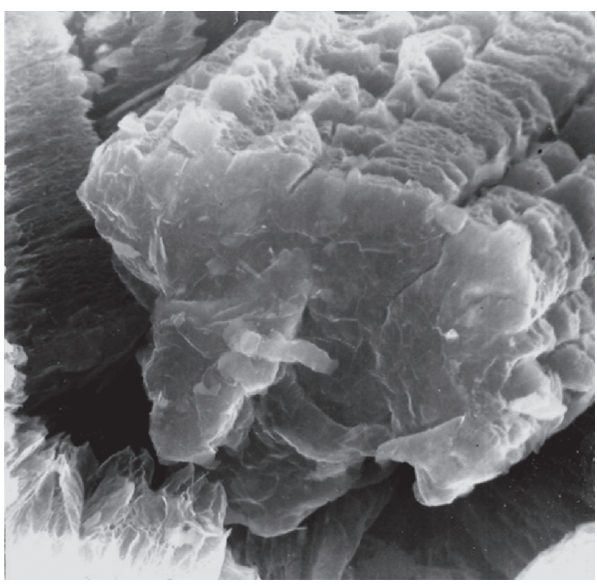

(b)

FIGURE 15: The layered compound of graphite in the initial state (a) and after the thermal shock (b) $(\times 30)$.

cannot maintain the pearlite transformation for a long time. At lower degrees of supercooling, which take place during cooling in graphite or by the furnace, heat loss to the surrounding medium decreases, and therefore conditions to increase the duration of the transformation are created. During normalization in the surface layer, the pearlite transformation occurs at a lower temperature than in the central part, the structure in the surface layer should be smaller, and larger in the central part. With induction heating, the product will only warm up to a certain depth, so heat removal will proceed more intensively and the conversion time will decrease more. In the case of cooling in graphite, the pearlite transformation starts at a temperature by $30^{\circ} \mathrm{C}$ higher than when cooled in air but ends at the same temperature. It follows from this that after completion of the pearlite transformation the structure of the samples after cooling in graphite should be more uniform. Investigations were carried out on deposited steel C45 samples with a diameter of $40 \mathrm{~mm}$ by the steel $\mathrm{C} 45$ tape (welding mode: $\left.I=6 \mathrm{kA}, P=1.25 \mathrm{kN}, t_{\mathrm{imp}}=0.04 \mathrm{sec}\right)$ after induction heating with high-frequency currents (heating mode: heating time
$35 \mathrm{~s}$. power density $1.8 \cdot 106 \mathrm{~W} / \mathrm{m}^{2}$ ). The length of the deposited sample is $50 \mathrm{~mm}$ and the diameter is $55 \mathrm{~mm}$. The samples were cooled in still air $\left(19^{\circ} \mathrm{C}\right)$ and in a heat-insulating medium, which contains the following parts: GL1 silver granular graphite GOST 5279-74 with a technological impurity of low-temperature compound intercalated graphite, which has the appearance of black crystalline powder (Figures 15 and 16).

The low-temperature compound of intercalated graphite with the general formula $\mathrm{CnO}$ was obtained from the natural graphite of the GT-2 brand in the following way [18]. The graphite powder was poured into the anode space of the electrolyzer, separated from the cathode space by a glass diaphragm, compressing it to a density exceeding 1.3-1.5 times the density of the original powder. A graphite current lead was placed on the surface of the compacted powder, on which a load was placed to improve electrical contact between the particles of graphite. An electrolyte was poured into the cathode space of the electrolyzer.

After wetting, direct current was passed through the electrolyzer. Electrolysis was carried out at a temperature not 


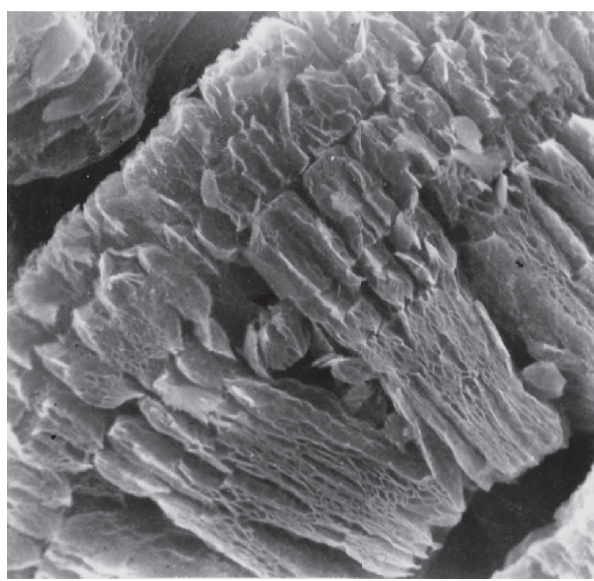

(a)

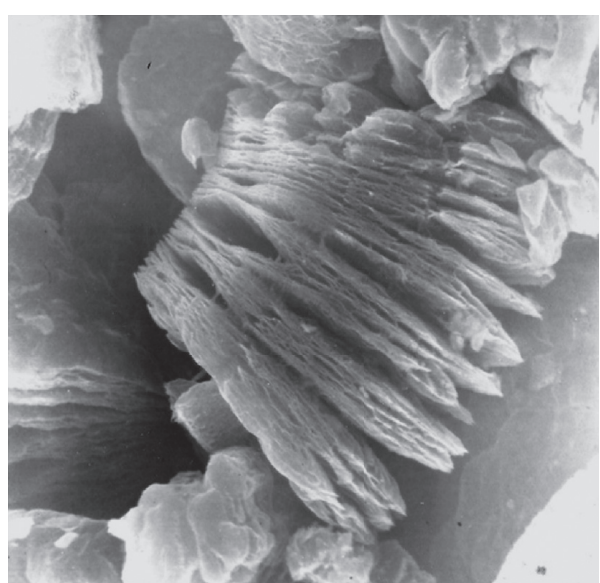

(b)

FIGURE 16: Graphite layered compound after the thermal shock $(\times 60)(a)$ and $(\times 100)(b)$.

higher than $30^{\circ} \mathrm{C}$ for the time required to reduce the initial current of the electrolyzer by $90-95 \%$. The resulting mass was taken out of the cell, washed thoroughly until the water did not contain acid (its concentration was determined by the weight method of $\mathrm{BaOH})$.

The obtained low-temperature compound of intercalated graphite has the following characteristics: bulk density $0.3-0.4 \mathrm{~g} / \mathrm{cm}^{3}$, the temperature of effective expansion $200-250^{\circ} \mathrm{C}$, and coefficient of thermal expansion $150-170 \mathrm{~cm}^{3} / \mathrm{g}$. When heated to $200^{\circ} \mathrm{C}$, thermal decomposition of intercalated graphite to penografit occurs (see Figures 15 and 16), which has an assumed density of $0.002 \pm 0.001 \mathrm{~g} / \mathrm{cm}^{3}$. Penografit is formed as a result of the graphite crystals separation in the interlayer spatial products of the polyarene layers of oxygen complexes pyrolysis [19]. The thermal expansion coefficient was determined as the ratio of the expanded low-temperature intercalated graphite compound volume after heating to a given temperature $\left(\mathrm{cm}^{3}\right)$ by weight of the initial sample $(\mathrm{g})$. For this purpose, a canopy of synthesized low-temperature intercalated graphite compound was introduced into the upper hole of a vertical tubular furnace, which, as the volume increased, entered the cylinder through the lower opening of the furnace to measure the volume. The nature of the covering agent heating when the deposited samples are in it after heating by high-frequency currents was fixed using a thermocouple TCA, located at a distance of $10 \mathrm{~mm}$ from the deposited surface at the level of the middle of its length. It was established experimentally that when using graphite oxide in an amount of $8-12$ wt.\%, the mixture heat did not exceed $30^{\circ} \mathrm{C}$, while when using $100 \%$ silver-lined graphite GL-1, heating reaches $240^{\circ} \mathrm{C}$ during the same time [17]. It was established that with a smaller amount of graphite oxide in the mixture, the protective effect is not significant, and with a content of more than $12 \mathrm{wt}$ \%, it is technologically inefficient [20]. Thus, the use of the developed covering agent reduces the energy consumption during the heat treatment of the deposited samples, increasing the effect of thermal insulation as it approaches the processing conditions in the furnace (Figure 17).

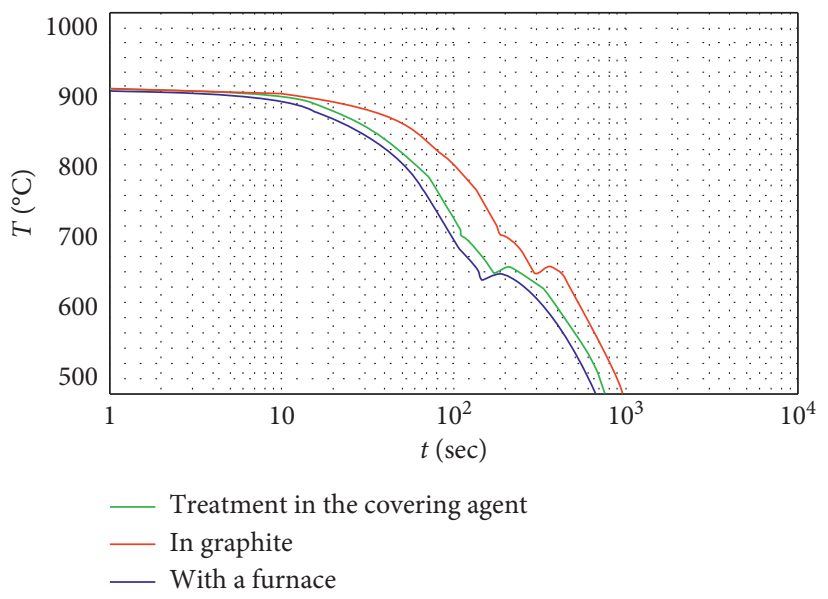

Figure 17: Temperature change of deposited steel C45 samples with a diameter of $20 \mathrm{~mm}$ when exposed to a covering agent as compared with standard cooling methods.

After the combined treatment, to ensure the specified dimensional accuracy, the overlaid samples were polished by a circular grinding machine with a 14A40SM2K5 brand PP300 $\times 25$ grinding wheel at the mode: lap speed $35 \mathrm{~m} / \mathrm{s}$, the circumferential speed of the deposited part is $35 \mathrm{~m} / \mathrm{min}$, and the radial feed rate is $0.001 \mathrm{~mm} / \mathrm{rev}$. $2 \%$ Acquol- 6 emulsion solution was used for cooling, and irrigation was supplied with a volume flow rate of $10 \mathrm{l} / \mathrm{min}$. [21]. The microstructure of samples subjected to normalization with air cooling and in a covering agent is shown in Figure 18.

As a result of quantitative metallographic analysis of various fields of view along the deposited samples, it was found that the exposure of the sample deposited by the electric contact method after high-frequency treatment in the covering agent (Figure 19) provided an increase in the content of excess ferrite [20].

Thus, the total concentration of excess ferrite in the deposited sample after treatment with high-frequency currents and exposure to the covering agent is $33.5899 \%$ with a minimum concentration of $28.4167 \%$ and a 


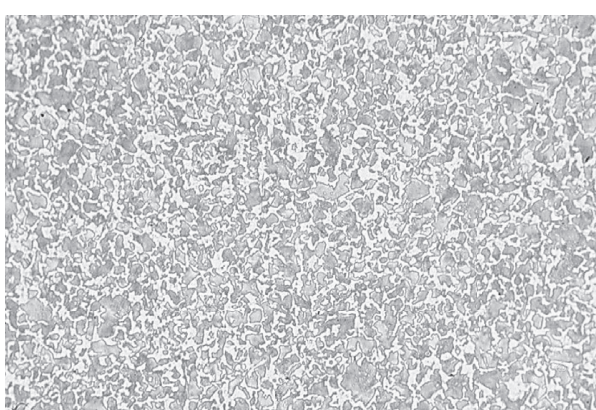

(a)

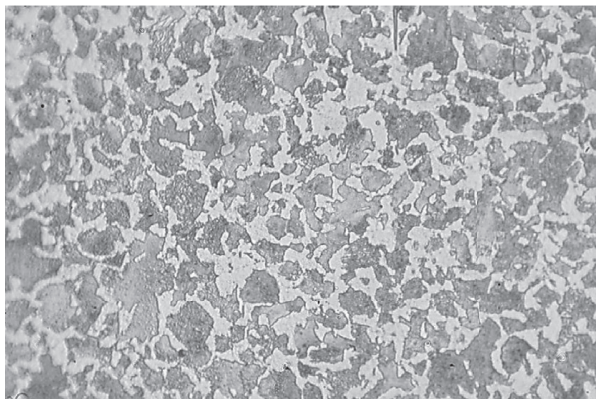

(c)

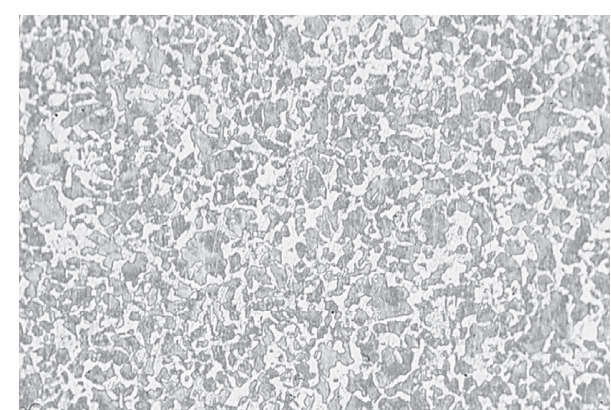

(b)

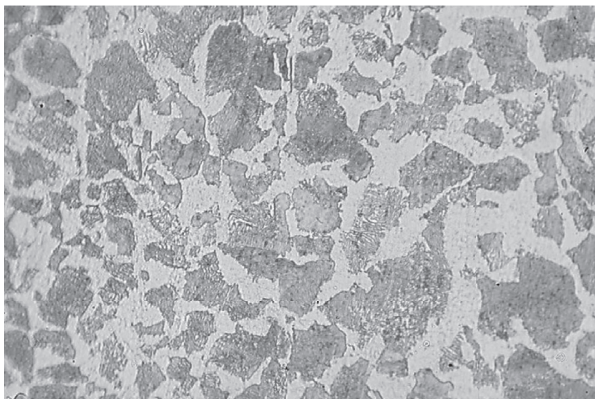

(d)

FIgURE 18: The microstructure of the deposited steel $\mathrm{C} 45$ sample after normalization from induction heating to $880^{\circ} \mathrm{C}$ in air $(\mathrm{a}, \mathrm{c})$ and in a covering agent $(b, d)$. (a) $\times 100$. (b) $\times 100$. (c) $\times 400$. (d) $\times 400$.

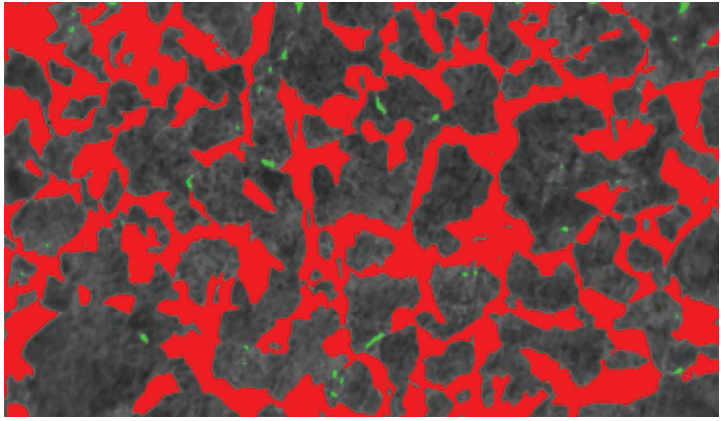

(a)

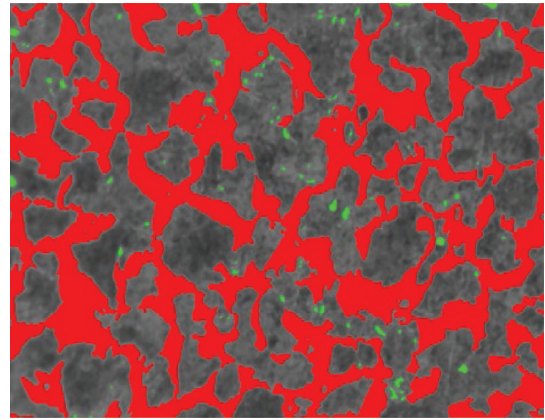

(c)

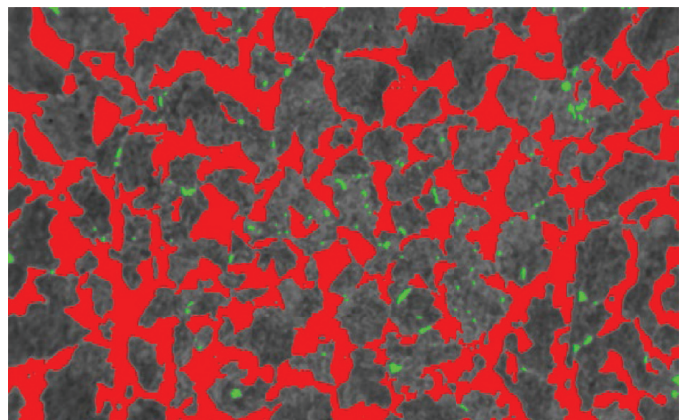

(b)

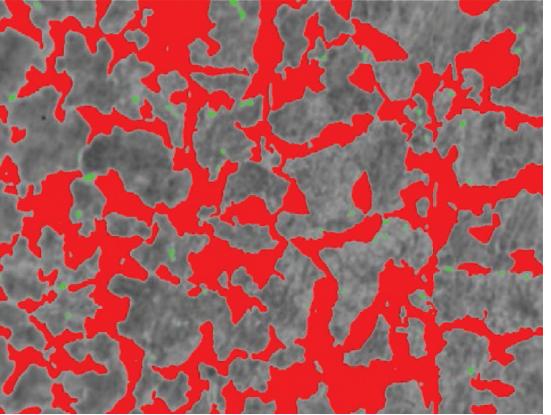

(d)

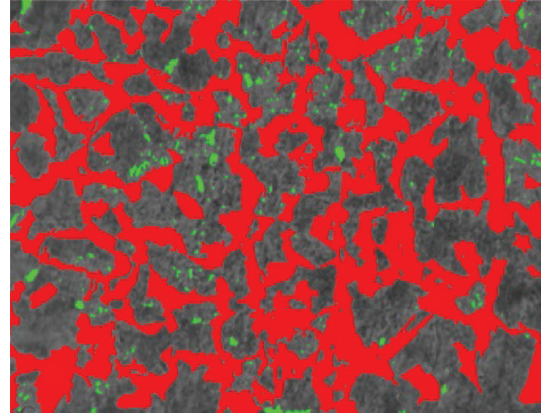

(e)

FIGURE 19: Quantitative analysis of structural elements when exposed into a covering agent in the field of view. № 1-excess ferrite concentration of $32.6751 \%$ (a); № 2-excess ferrite concentration of 33.5124\% (b); № 3-excess ferrite concentration of 35.7860\% (c); № 4-excess ferrite concentration of $33.7661 \%$ (d); № 5-the concentration of excess ferrite $37.7756 \%$ (e) $(\times 400)$. 


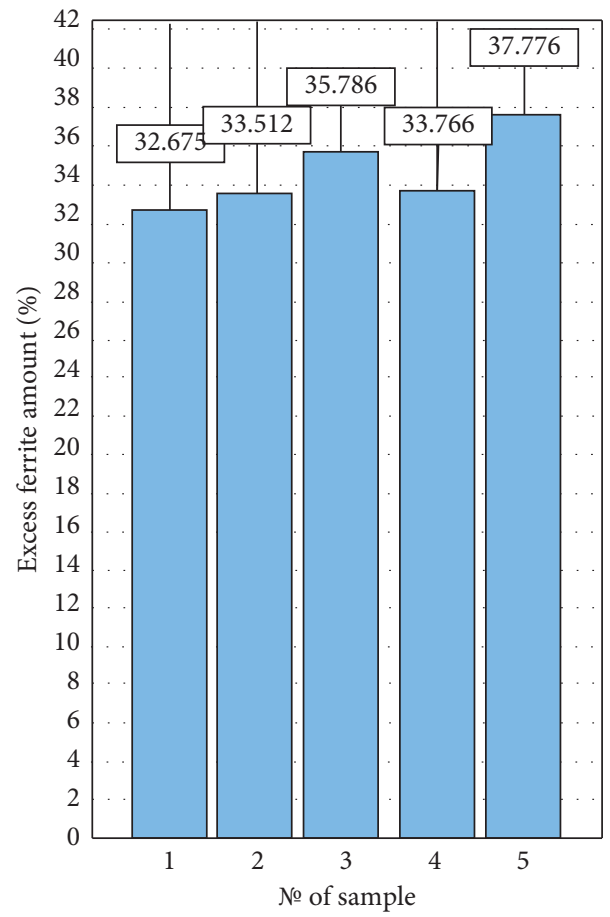

Figure 20: The distribution of excess ferrite amount in the deposited steel C45 samples after treatment with high-frequency currents and normalization in the covering agent.

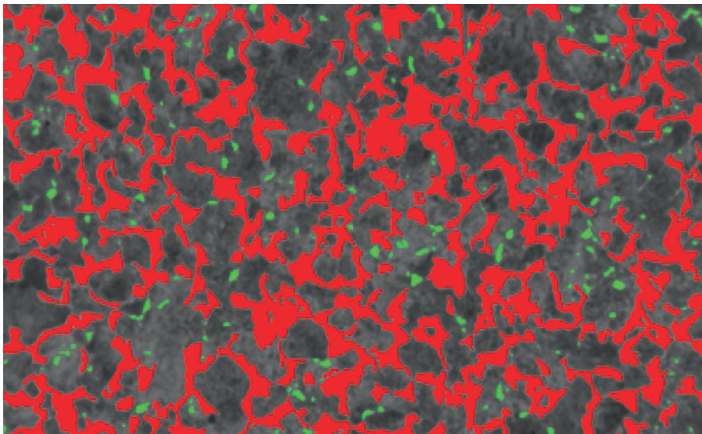

(a)

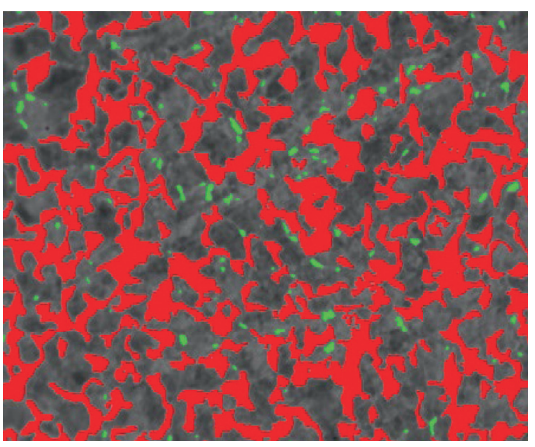

(c)

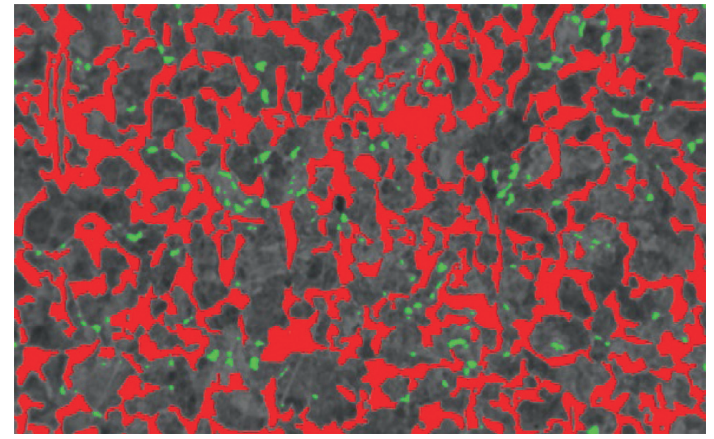

(b)

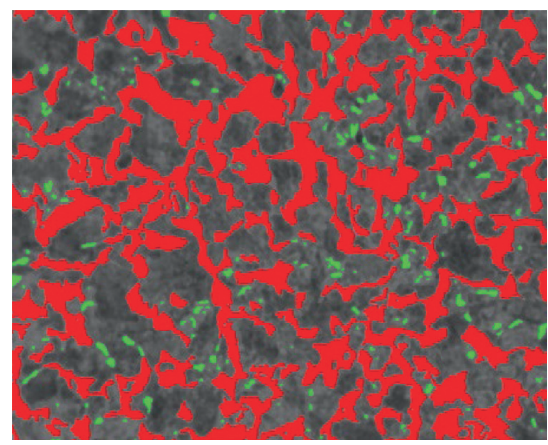

(d)

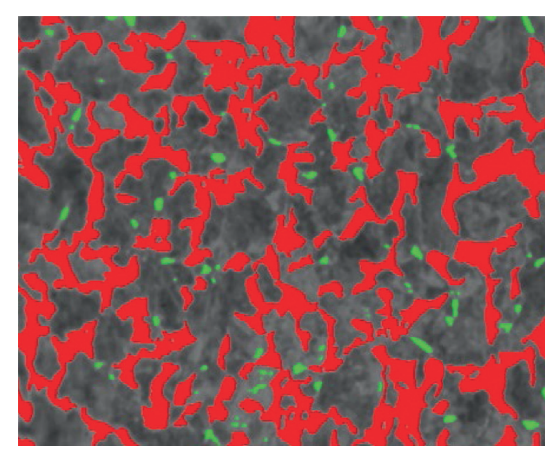

(e)

Figure 21: Quantitative analysis of structural elements during air cooling in the field of view. № 1-the concentration of excess ferrite is $28.8504 \%$ (a); № 2- excess ferrite concentration of 29.9103\% (b); № 3-excess ferrite concentration of 31.2454\% (c); № 4- excess ferrite concentration of $31.4373 \%$ (d); № 5-the concentration of excess ferrite is $28.5952 \%$ (e) $(\times 400)$. 
maximum of $37.7756 \%$ [20]. The results are presented in Figure 20.

The average content of excess ferrite during cooling of the samples deposited by the electrocontact method in air is, on average, $4.7 \%$ lower (Figures 21 and 22), as compared to the cooling of the deposited samples in the covering agent (Figure 23). Thus, the total concentration of excess ferrite in the deposited sample after treatment with high-frequency currents and air cooling is $30.0077 \%$ with a minimum concentration of excess ferrite in the amount of $28.5952 \%$ and a maximum of $31.4373 \%$. At the same time, the minimum concentration of excess ferrite in the samples after the combined recovery technology, including electrocontact surfacing, local processing by high-frequency currents, and cooling in the heat-insulating mixture is $32.675 \%$.

According to the research results, the average grain size of the deposited layer structure obtained by slow cooling is by $11 \mathrm{~mm}$ larger than the grain obtained by cooling the deposited samples in air (Tables 15-18). With different variations in the chemical composition of the deposited and base metal, the choice of combined treatment modes after electrical contact welding should be made on the basis of the material tendency to stress concentration.

\section{Discussion}

The experimentally established stress values that cause the first acts of microplastic deformation determine the average required stress value for the dislocations start, which causes the microplastic deformation. In the deposited samples, which are insensitive to stress concentration under cyclic loading conditions, the necessary average stress value for the start of dislocations is small. Thus, the smaller the magnitude of the stresses required to initiate the dislocations movement, the less sensitive the sample is to the stress concentration. The highest tendency to microplastic deformation is observed in the deposited steel C25 samples, the main structural part of which is ferrite. This is caused by the highest tendency to dislocation movement under the action of applied stresses. In these samples, deformation occurs the most easily, and relatively small stresses are required for both the start and the movement of dislocations.

The highest resistance of microplastic deformation is demonstrated by the deposited samples after thermal improvement. For example, for deposited 530H40 steel samples, improved for sorbitol tempering (group II), the required average stresses are $373 \mathrm{MPa}$ to cause a residual microplastic $4 \%$ deformation, and in these same samples with temposite tempering (group III)-497 MPa. Consequently, thermal improvement, causing the structure refinement, conditions an increased and even high resistance to microplastic deformation or dislocations movement. This may be caused by the significant number of obstacles to the dislocations movement.

Consequently, samples in a thermoimproved state also have the highest sensitivity to stress concentration under cyclic loading conditions.

In the course of the research, a technology for the worn parts combined treatment was proposed and a covering

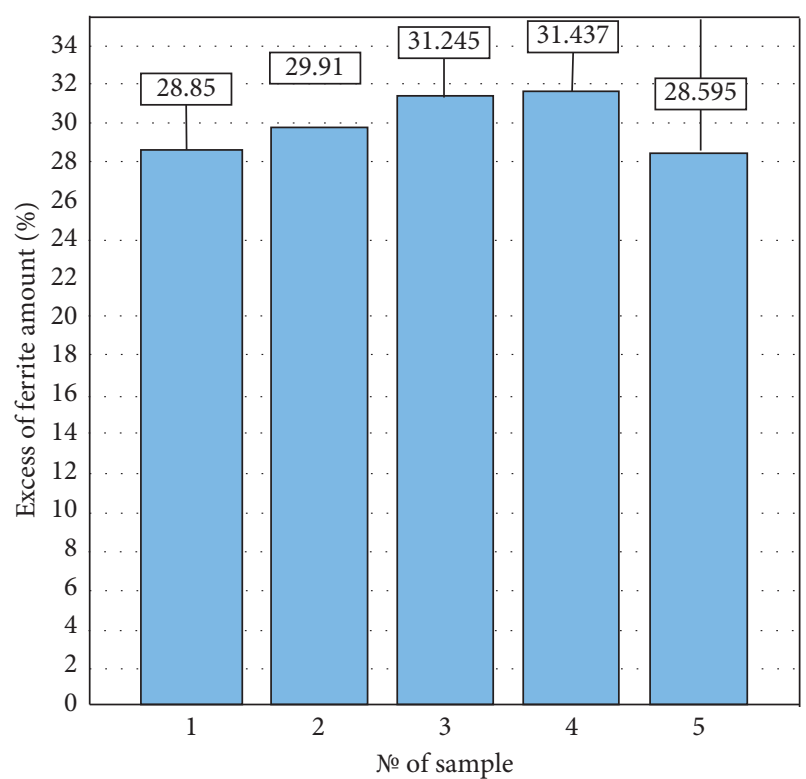

FIgURE 22: The distribution of the excess ferrite amount in the deposited steel $\mathrm{C} 45$ samples after treatment with high-frequency currents and air cooling.

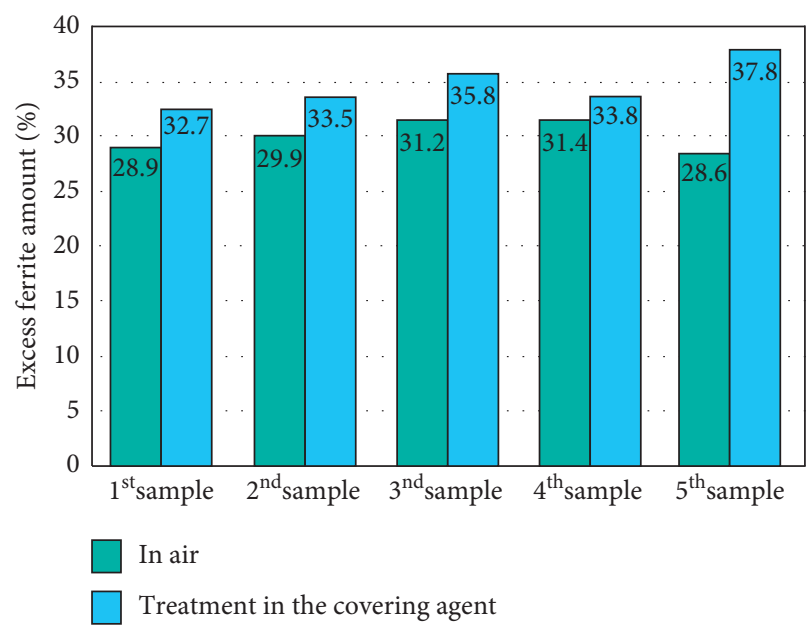

FIgURE 23: The distribution of the excess ferrite amount in the deposited steel C45 samples after cooling in air and cooling in the covering agent.

agent composition for slow cooling of the deposited samples was developed. The obtained results of the excess ferrite distribution in the deposited samples after treatment by high-frequency currents and cooling in air indicate a decrease of this indicator by 1.12 times as compared to the samples after the combined recovery technology (see Figure 22). This tendency persists in the quantitative metallographic analysis of all the deposited samples in different fields of view (see Figure 23). As follows from the grain sizes studies results, slow cooling during covering agent soak also leads to an increase in grain sizes.

Such a change in the grain size in the samples structure deposited by the electrocontact method is caused by the fact 
TABLE 15: Indicators of deposited steel C45 samples grain sizes after treatment with high-frequency currents when cooled in air.

\begin{tabular}{|c|c|c|c|c|c|}
\hline № & Type & Min & Max & Amount & $\%$ \\
\hline 1 & -3 & 723.6010 & 1023.3270 & 0 & 0.000 \\
\hline 2 & -2 & 511.6630 & 723.6010 & 0 & 0.000 \\
\hline 3 & -1 & 361.8010 & 511.6630 & 0 & 0.000 \\
\hline 4 & 0 & 255.8320 & 361.8010 & 0 & 0.000 \\
\hline 5 & 1 & 180.9000 & 255.8320 & 0 & 0.000 \\
\hline 6 & 2 & 127.9160 & 180.9000 & 0 & 0.000 \\
\hline 7 & 3 & 90.4500 & 127.9160 & 0 & 0.000 \\
\hline 8 & 4 & 63.9580 & 90.4500 & 0 & 0.000 \\
\hline 9 & 5 & 45.2250 & 63.9580 & 0 & 0.000 \\
\hline 10 & 6 & 31.9790 & 45.2250 & 0 & 0.000 \\
\hline 11 & 7 & 22.6130 & 31.9790 & 3 & 5.000 \\
\hline 12 & 8 & 15.9890 & 22.6130 & 13 & 21.667 \\
\hline 13 & 9 & 11.3060 & 15.9890 & 29 & 48.333 \\
\hline 14 & 10 & 7.9950 & 11.3060 & 13 & 21.667 \\
\hline 15 & 11 & 5.6530 & 7.9950 & 2 & 3.333 \\
\hline 16 & 12 & 3.9970 & 5.6530 & 0 & 0.000 \\
\hline 17 & 13 & 2.8270 & 3.9970 & 0 & 0.000 \\
\hline 18 & 14 & 1.9990 & 2.8270 & 0 & 0.000 \\
\hline
\end{tabular}

TABLE 16: Indicators of deposited steel C45 samples grain sizes of steel C45 processed by high-frequency current treatment during cooling in air.

\begin{tabular}{lcccc}
\hline Parameter & Medium & Shift & Minimum & Maximum \\
\hline Length $(\mathrm{mm})$ & 14.25 & 0.52 & 7.16 & 25.55 \\
\hline
\end{tabular}

TABLE 17: Indicators of deposited steel C45 samples grain sizes after treatment by high-frequency currents and air cooling.

\begin{tabular}{lcccc}
\hline Parameter & Medium & Shift & Minimum & Maximum \\
\hline Length $(\mathrm{mm})$ & 25.21 & 0.85 & 11.92 & 51.48 \\
\hline
\end{tabular}

TABLE 18: Indicators of deposited steel C45 samples grain sizes after treatment with high-frequency currents and air cooling.

\begin{tabular}{|c|c|c|c|c|c|}
\hline № & Type & Min & Max & Amount & $\%$ \\
\hline 1 & 2 & 3 & 4 & 5 & 6 \\
\hline 1 & -3 & 723.6010 & 1023.3270 & 0 & 0.000 \\
\hline 2 & -2 & 511.6630 & 723.6010 & 0 & 0.000 \\
\hline 3 & -1 & 361.8010 & 511.6630 & 0 & 0.000 \\
\hline 4 & 0 & 255.8320 & 361.8010 & 0 & 0.000 \\
\hline 5 & 1 & 180.9000 & 255.8320 & 0 & 0.000 \\
\hline 6 & 2 & 127.9160 & 180.9000 & 0 & 0.000 \\
\hline 7 & 3 & 90.4500 & 127.9160 & 0 & 0.000 \\
\hline 8 & 4 & 63.9580 & 90.4500 & 0 & 0.000 \\
\hline 9 & 5 & 45.2250 & 63.9580 & 4 & 4.167 \\
\hline 10 & 6 & 31.9790 & 45.2250 & 13 & 13.542 \\
\hline 11 & 7 & 22.6130 & 31.9790 & 34 & 35.417 \\
\hline 12 & 8 & 15.9890 & 22.6130 & 36 & 37.500 \\
\hline 13 & 9 & 11.3060 & 15.9890 & 9 & 9.375 \\
\hline 14 & 10 & 7.9950 & 11.3060 & 0 & 0.000 \\
\hline 15 & 11 & 5.6530 & 7.9950 & 0 & 0.000 \\
\hline 16 & 12 & 3.9970 & 5.6530 & 0 & 0.000 \\
\hline 17 & 13 & 2.8270 & 3.9970 & 0 & 0.000 \\
\hline 18 & 14 & 1.9990 & 2.8270 & 0 & 0.000 \\
\hline
\end{tabular}


that the diffusion transformation of austenite into a ferritecementite mixture during cooling in the covering agent proceeds more slowly and at higher temperatures.

Besides, there is a general increase in grain size in the structure of the deposited samples after the combined restoration technology, which contributes to reducing the number of obstacles to the dislocations movement when operating under cyclic loading, and consequently, to increase the fatigue strength of the restored parts.

\section{Conclusion}

The structural factor of samples microplasticity deposited by tapes of carbon-bearing structural steels was investigated. It was found that the highest resistance of microplastic deformation occurs in the deposited samples after their heat treatment with the formation of sorbitol and troostite due to the developed intergranular surface and an increase in the number of obstacles for dislocation slip. This causes higher starting stresses (for group II)-59 MPa; for group III-201 MPa). The deposited samples containing a larger volume of excess ferrite grains are distinguished by a smaller number of obstacles for dislocation shear, and the starting stresses in the samples are $30.5 \mathrm{MPa}$, which indicates a low sensitivity of the deposited layer to the stress concentration.

The highest resistance of microplastic deformation is demonstrated by the deposited samples after thermal improvement. For example, for deposited 530H40 steel samples, improved for sorbitol tempering (group II), the required average stresses are $373 \mathrm{MPa}$ to cause a residual microplastic $4 \%$ deformation, and in these same samples with temposite tempering (group III)-497 MPa. Consequently, thermal improvement, causing the structure refinement, conditions an increased and even high resistance to microplastic deformation or dislocations movement. This may be caused by the significant number of obstacles to the dislocations movement. Consequently, samples in a thermoimproved state also have the highest sensitivity to stress concentration under cyclic loading conditions.

In the course of the research, a technology for the worn parts combined treatment was proposed and a covering agent composition for slow cooling of the deposited samples was developed. It has been experimentally established that the use of a heat-insulating mixture of the developed composition as a cooling medium during the heat treatment of deposited parts makes it possible to bring the cooling conditions closer to the furnace ones. Application of the developed combined technology for restoring parts operating under cyclic loading (electrical contact welding with subsequent local heating by high-frequency currents up to $900^{\circ} \mathrm{C}$ and slow cooling in the mixture-8-12\% layered graphite compound with a thermal expansion coefficient of $150-170 \mathrm{~cm}^{3} / \mathrm{g}$ at a temperature of $200-250^{\circ} \mathrm{C}$, the rest is graphite GL1) reduces the cooling rate from the austenitization temperature to the onset of pearlite transformation and increases the average content of excess ferrite by $4.7 \%$ with an increase in the average grain size by $11 \mathrm{~mm}$. For parts operating under cyclic loading conditions, such a structure is the least sensitive to stress concentration.

\section{Data Availability}

Data sharing is not applicable to this article as no datasets were generated or analyzed during the current study.

\section{Conflicts of Interest}

The authors Olena V. Berezshnaya, Valeriy D. Kassov, and Eduard P. Gribkov declare that there are no conflicts of interest regarding the publication of this paper.

\section{Authors' Contributions}

All authors participated in the design of this work and performed equally. All authors read and approved the final manuscript.

\section{References}

[1] P. I. Burak, A. V. Serov, and R. A. Latypov, "Optimization of the process of electric resistance welding of metallic strips through an amorphous solder," Welding International, vol. 26, no. 10, pp. 814-818, 2012.

[2] R. N. Sayfullin and K. V. Yuferov, "The microstructure of coatings on high-carbon steel strips produced by electric resistance deposition," Welding International, vol. 27, no. 9, pp. 717-719, 2013.

[3] R. N. Sayfullin, M. N. Farkhshatov, V. S. Natalenko, and N. M. Yunusbaev, "Distribution of microhardness in coatings and its effect on resistance," Uprochnyayushchie Tekhnologii I Pokrytiya, vol. 2, no. 26, pp. 18-21, 2007, in Russian.

[4] P. I. Burak, "Driving forces of cohesion and formation of surface bonding process in electric resistance welding-on," International Technological and Economic Journal, vol. 4, no. 4, pp. 33-37, 2007.

[5] M. D. Chapetti, T. Tagawa, and T. Miyata, "Ultra-long cycle fatigue of high-strength carbon steels. Part 1: revive and analysis of the mechanism of failure," Materials Science and Engineering, vol. 356, no. 1-2, pp. 227-235, 2003.

[6] L. I. Markashova, V. D. Poznyakov, E. N. Berdnikova et al., "Effect of structural factors on mechanical properties and crack resistance of welded joints of metals, alloys and composite materials," The Paton Welding Journal, vol. 6, no. 7, pp. 22-28, 2014.

[7] A. A. Gajvoronsky, V. D. Poznyakov, L. I. Markashova et al., "Influence of deposited metal composition on structure and mechanical properties of reconditioned railway wheels," The Paton Welding Journal, vol. 8, pp. 16-22, 2012.

[8] L. I. Markashova, V. V. Arsenyuk, and G. M. Grigorenko, "Relationships governing plastic deformation in pressure welding dissimilar materials," Welding International, vol. 19, no. 1, pp. 68-72, 2005.

[9] K. V. Yuferov, "Special features of electric resistance deposition of the heat-treated steel strip," Remont, Vosstanovlenie, Modernizatsiya, vol. 1, pp. 23-25, 2012, in Russian.

[10] O. V. Berezshnaya, V. D. Kuznetsov, V. D. Kassov, and P. A. Gavrish, "Investigation of microplastic deformation of metal deposited by electric contact method," Automatic Welding, vol. 2017, no. 7, pp. 24-28, 2017, in Russian.

[11] S. A. Madyanov, V. R. Kalinin, and A. P. Kraev, "Examination of microplastic deformation as a method of evaluation of metal embrittlement," Mechanics and Physics of Fracture of Brittle Materials, vol. 2, no. 1, pp. 33-38, 1990. 
[12] P. I. Burak and R. A. Latypov, "Effect of the intermediate layer on the properties of a coating of $50 \mathrm{KhFA}$ steel produced by electrical resistance welding," Welding International, vol. 26, no. 7, pp. 547-549, 2012.

[13] E. Berezshnaya, J. Chepel, N. Tsyvinda, and A. Pikilnyak, "Mathematic modeling of detail's restoration combined process," Metallurgical and Mining Industry, vol. 7, no. 10, pp. 198-201, 2015.

[14] G. F. Golovin and M. M. Zamyatin, High-Frequency Heat Treatment, Mashinostroenie, Leningrad, Russia, in Russian, 1990.

[15] A. Vasiliev, I. Pozniak, and V. Greshnov, "Modeling and investigation of hardening processes," in Proceedings of the International Scientific Colloquium Modeling for Electromagnetic Processing, pp. 24-26, Hannover, Germany, March 2003.

[16] M. L. Bernshtein and A. G. Rakhshtadt, Metal Science and Heat Treatment of Steel: A Handbook, vol. 2, 1983 in Russian.

[17] V. K. Zablotsky and V. I. Shimko, "Features of structuralphase transformations in $45 \mathrm{Kh} 3 \mathrm{GNMF}$ steel under continuous cooling and in isothermal conditions," Stroitelstvo. Materialovedenie. Mashinostroenie, vol. 58, pp. 292-294, 2011, in Russian.

[18] A. V. Likov, Theory of Heat Conductivity, Moscow: High School, Moscow, Russia, 1967, in Russian.

[19] M. V. Savoskin, V. N. Mochalin, A. P. Yaroshenko et al., "Carbon nanoscrolls produced from acceptor-type graphite intercalation compounds," Carbon, vol. 45, no. 14, pp. 2797-2800, 2007.

[20] E. V. Berezshnaya, "Technological features of heat treatment of parts restored by electrical contact surfacing," Actual Problems in Machine Building, vol. 4, no. 1, pp. 126-131, 2017, in Russian.

[21] E. V. Berezshnaya, "The choice of induction heating to normalize the weld surfaces of cylindrical parts," Reporter of the Priazovskyi State Technical University, vol. 34, pp. 73-80, 2017, in Russian. 